\title{
Surgery and Rehabilitation Treatment Options for Ulnar Collateral Ligament Injuries of the Elbow for Baseball Athletes: A Systematic Review
}

Amanda M. Damm

Follow this and additional works at: https://researchrepository.wvu.edu/etd

\section{Recommended Citation}

Damm, Amanda M., "Surgery and Rehabilitation Treatment Options for Ulnar Collateral Ligament Injuries of the Elbow for Baseball Athletes: A Systematic Review" (2015). Graduate Theses, Dissertations, and Problem Reports. 5432.

https://researchrepository.wvu.edu/etd/5432

This Thesis is protected by copyright and/or related rights. It has been brought to you by the The Research Repository @WVU with permission from the rights-holder(s). You are free to use this Thesis in any way that is permitted by the copyright and related rights legislation that applies to your use. For other uses you must obtain permission from the rights-holder(s) directly, unless additional rights are indicated by a Creative Commons license in the record and/ or on the work itself. This Thesis has been accepted for inclusion in WVU Graduate Theses, Dissertations, and Problem Reports collection by an authorized administrator of The Research Repository @ WVU. For more information, please contact researchrepository@mail.wvu.edu. 
Surgery and Rehabilitation Treatment Options for Ulnar Collateral Ligament Injuries of the Elbow for Baseball Athletes: A Systematic Review

\author{
Amanda M. Damm, ATC \\ Thesis submitted to the \\ College of Physical Activity and Sport Science \\ at West Virginia University \\ in partial fulfillment of the requirements \\ for the degree of \\ Master of Science \\ In \\ Athletic Training \\ Michelle A. Sandrey, Ph.D., ATC, Chair \\ Jordan Feathers, MPT, OCS, CSCS \\ Jean McCrory, Ph.D.
}

Department of Sports Sciences

Morgantown, WV

2015

Key Words: ulnar collateral ligament, elbow, baseball, reconstruction, treatment 


\begin{abstract}
Surgery and Rehabilitation Treatment Options for Ulnar Collateral Ligament Injuries of the Elbow for Baseball Athletes:

A Systematic Review
\end{abstract}

Amanda M. Damm, BS, ATC

Context: There is currently a lack of information on ulnar collateral ligament (UCL) injuries for athletic trainers. Most of the literature addressed the surgical options in detail and depending on the study, information for rehabilitation may be limited or not even included. Further, there are a limited number of studies that compare the different surgical techniques to each other as well. Objective: To provide a comparison of the Jobe technique to the Docking technique, in regard to surgery, success rates, and rehabilitation process. Data Sources: PubMed (1960-2014), MEDLINE (1960-2014), CINAHL with full text (1960-2014), SPORTDiscus full text (19602014), Science Direct (1960-2014), and Google Scholar were searched using the terms ulnar collateral ligament, elbow, baseball, reconstruction and treatment. Second, the term ulnar collateral ligament was combined with each of the following terms: surgery, Jobe technique, Docking technique, and rehabilitation. Third, cross-referenced citations were used from studies that included information not found in searches. Study Selection: Studies were included based on the following criteria: 1) written in English or an English translation; 2) ulnar collateral ligament, baseball athletes, throwing athletes, surgical techniques, elbow, reconstruction, rehabilitation, or various forms of these terms were mentioned in the title of the study; 3) ulnar collateral ligament, baseball athletes, throwing athletes, surgical techniques, elbow, reconstruction, rehabilitation, or various forms of these terms were mentioned in the abstract; 4) ulnar Collateral ligament reconstruction and rehabilitation was the main focus of the study; 5) the study was an experimental study or cohort study. Studies were excluded on the following criteria: 1) they are not written in or translated into English; 2) studies do not involve the Jobe or Docking technique as a surgical option; 3) injury did not pertain to the UCL of the elbow. Data Extraction: All studies that met the inclusion criteria were evaluated using the Coleman Methodology Score (CMS). Each study included was read first by each evaluator without the Coleman Methodology Score. The studies included were read a second time using the Coleman Methodology Score. Final score was within a range of 0 to 100 . Once all studies have were read and evaluated, the evaluators' met to compare scores. When discrepancies were found, the evaluators' discussed the criterion in question and agreed on a final score. Data Synthesis: Twelve studies met the inclusion criteria and were evaluated with the Coleman Methodology Score. The methodology scores ranged from 37-62, average score was 49.2 out of 100 . Six studies scored fair and 6 studies scored poor on the CMS. Success between surgical techniques was inconclusive for all surgical techniques examined based on CMS scores. Conclusion: The CMS scores ranged from fair to poor. It can be concluded that no recommendations can be made on what surgical technique is considered to be the best options for UCL reconstruction and that results may remain inconclusive. 


\section{ACKNOWLEDGEMENTS}

To my family who has been there supporting me throughout all the years and have done nothing but encouraged all of my dreams and goals. They have been my support system from day one and I do not think I would be where I am today without all of their love, support and encouragement to be the best I can be.

To the athletic department staff at Waynesburg Central High School, although times started out rough in the beginning, I am happy to say that I have learned so much being able to work at their high school as an Athletic Trainer. The athletes, coaches and athletic director all helped in encouraging me to be the best I can be.

To Jordan Feathers and Dr. Jean McCrory for being on my thesis committee. Thank you for all of your time and effort put into making my thesis the best it can be. They all have provided me with new ways to think about this topic and approach the injury from a clinical perspective as well as research in terms of critical thinking.

A special thanks goes out to my Western Michigan University family, thank you all of your assistance and support over the years. I do not think I would be where I am today or the athletic trainer I am today without the two wonderful women who run the program, Dr. Gayle Thompson and Gretchen Mohney. They have always been there for me ever since I started my journey in the Athletic Training Professional Program and continue to show their support over the years.

To my friends and fellow classmates, for all of their support and understanding of the past two stressful years.

And finally to Dr. Sandrey, for her guidance throughout this process and the countless hours spent in meetings and providing valuable feedback on my drafts. I greatly appreciate all that you have done for me and for this study over the past two years. 


\section{TABLE OF CONTENTS}

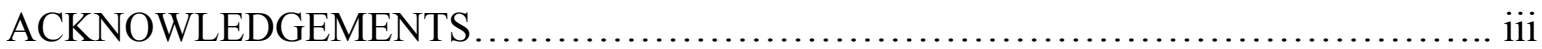

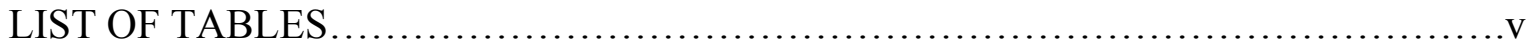

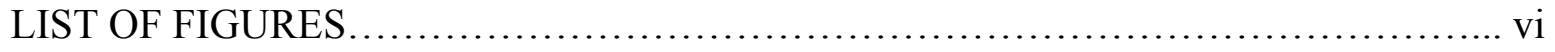

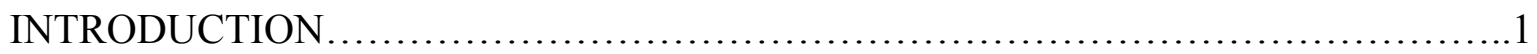

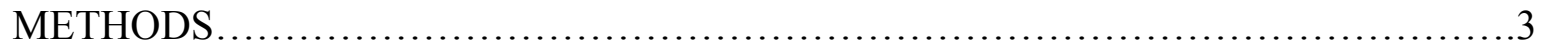

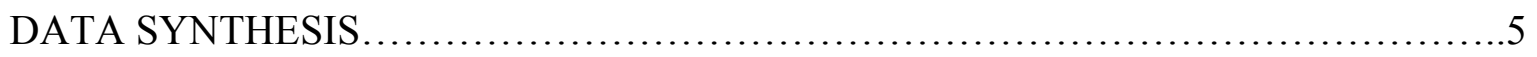

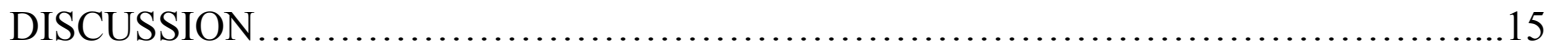

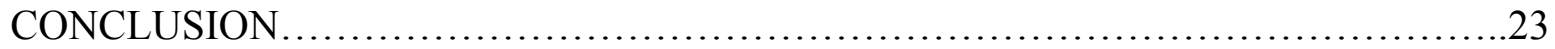

REFERENCES.............................................................. 24

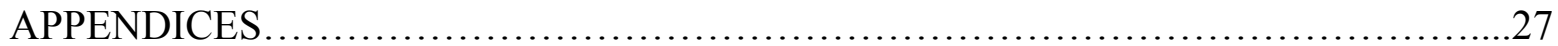

APPENDIX A. THE PROBLEM..........................................28

APPENDIX B. LITERATURE REVIEW ...................................36

APPENDIX C. ADDITIONAL METHODS...................................61

APPENDIX D. ADDITIONAL RESULTS ...................................65

APPENDIX E. RECOMMENDATIONS FOR FUTURE RESEARCH.............88

ADDITIONAL REFERENCES ...................................................... 89 
Table

\section{LIST OF TABLES}

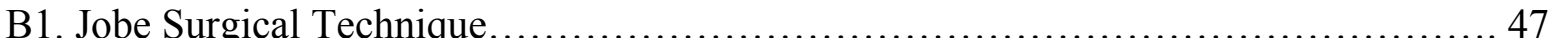

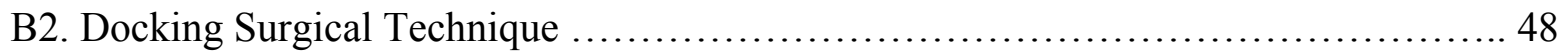

B3. Jobe Technique Rehabilitation.............................................. 54

B4. Docking Technique Rehabilitation.......................................... 56

C1. Inclusion/Exclusion Criteria............................................ 61

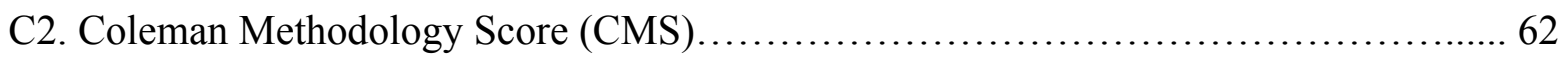

C3. Description of Coleman Methodology Score (CMS) ........................... 63

D1. Jobe Surgical Technique Studies........................................ 65

D2. Docking Surgical Technique Studies..................................... 66

D3. Coleman Methodology Score (CMS) Results............................. 68

D4. Azar FM et al. Coleman Methodology Score................................... 69

D5. Bowers AL et al. Coleman Methodology Score............................... 70

D6. Cain EL et al. Coleman Methodology Score..................................................... 71

D7. Dodson CC et al. Coleman Methodology Score................................................... 72

D8. Dugas JR et al. Coleman Methodology Score............................................................ 73

D9. Jobe FW et al. Coleman Methodology Score....................................................... 74

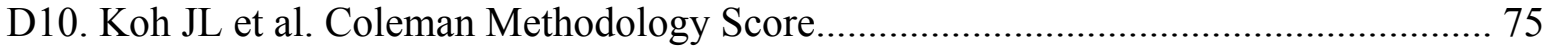

D11. Merolla G et al. Coleman Methodology Score................................................... 76

D12. Osbahr DC et al. Coleman Methodology Score................................................. 77

D13. Paletta GA et al. Coleman Methodology Score.................................................... 78

D14. Rohrbough JT et al. Coleman Methodology Score.............................................. 79

D15. Savoie FH et al. Coleman Methodology Score ................................................ 80 


\section{LIST OF FIGURES}

Figure Page

B1. Anatomy of Medial Elbow............................................. 37

B2. Jobe Technique...................................................... 44

B3. Docking Technique.................................................. 45

D1. PRISMA Flow Diagram-Google Scholar......................................................... 81

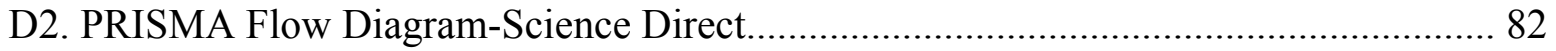

D3. PRISMA Flow Diagram-CINAHL with full text............................................ 83

D4. PRISMA Flow Diagram- MEDLINE (EbscoHost)............................................. 84

D5. PRISMA Flow Diagram- MEDLINE (PudMed)................................................. 85

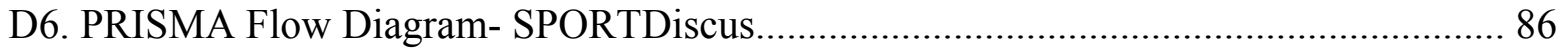

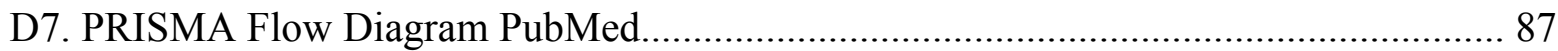




\section{INTRODUCTION}

Injuries sustained in baseball are quite common with the upper extremity accounting for $45 \%$ of those injuries. ${ }^{1}$ Injuries to the glenohumeral joint and sprains/strains are more evident, but injuries to the elbow follow closely. ${ }^{1,2}$ The most common injury to the elbow in baseball is a torn ulnar collateral ligament (UCL). Of the ligamentous structures that comprise the UCL, the anterior bundle, the primary stabilizer for the medial aspect of the elbow, is most commonly torn. ${ }^{3-12}$ It has been reported that about $26.3 \%$ of pitchers sustain an elbow injury within a season. ${ }^{2}$ There are various intrinsic and extrinsic factors that contribute to baseball athletes, specifically pitchers, sustaining injuries to the UCL. Pitchers are more likely to sustain an injury in a game as they throw through pain as well as pitch faster. ${ }^{1}$

The UCL is commonly found to tear during the late cocking/early acceleration phase of throwing. This is due to an excessive valgus force, which stresses the medial side of the elbow during these two phases of throwing. ${ }^{3-14}$ The nature of the injury is classified as a chronic injury because mircotears occur along the UCL during the throwing motion. ${ }^{8-10,12,14,15}$ However, there is a chance for an acute tear while throwing. ${ }^{5,69,12,14,15}$ The injury occurs due to biomechanical abnormalities at the glenohumeral or the elbow joints ${ }^{12,15,16}$ and throwing errors. ${ }^{17}$

Knowledge of the injury and the treatment options involved has increased over the years. Until the 1970 's, there were no surgical options available. ${ }^{8}$ If a baseball player sustained an injury to the UCL, a baseball career was considered to be over. In 1974, Dr. Frank Jobe changed the treatment options by creating a surgical technique in which the UCL was reconstructed. ${ }^{8}$ This provided hope for baseball players to continue with their career in baseball. According to Jobe et al., ${ }^{8}$ surgery should be the first line of defense and the best method to treat UCL injuries. However, some physicians suggested that the first line of defense in the treatment of UCL should be three months of rehabilitation before a surgical option is explored..$^{3,4,8-10}$ 
When a conservative approach fails, a surgical option is explored. Since the 1970's, numerous modifications evolved from the Jobe technique and other surgical techniques were created based on this original technique. ${ }^{8,9,18}$ A modified technique that is commonly used and has a higher success rate is the Docking technique. ${ }^{18-22}$ There are some notable differences and similarities between these two surgical techniques in the treatment of a torn UCL. The most common tendon graft used for both surgical techniques is the palmaris longus. ${ }^{3,6,8-10,12,19,21,22}$ The difference between the two techniques are the modifications that were made when the Docking technique was created. One notable difference between the two techniques is the avoidance of the transposition of the ulnar nerve during the Docking technique. ${ }^{21}$ The only time a surgeon will transpose the nerve is when it cannot be protected, ${ }^{20}$ or there is a history of ulnar neuropathy. ${ }^{22}$

The post-operative rehabilitation for a UCL reconstruction is deemed to be a fairly extensive process. The rehabilitation protocol is tailored to the technique used for the reconstruction and the attending physicians comfort with how early or late progressions occur. Like most rehabilitation protocols, specific goals are to be met throughout the process for an athlete to return to play. Some of these goals are for the athlete to regain full range of motion and strength at the elbow and glenohumeral joint and to be able to successfully throw through all the throwing phases pain free. ${ }^{6,8,9}$

There is an abundance of studies performed on the surgical treatment options of UCL injuries, the epidemiology and etiology of the injury, and outcome measures published by physicians and/or surgeons. However, limited information exists for allied health professionals that rehabilitate the pitcher following UCL reconstruction. Some studies may summarize what the rehabilitation protocol is in one-two paragraphs. This could be problematic due to the uncertainty on what might be expected during rehabilitation and also when progressions should be allowed. A systematic review is performed to evaluate the quality of studies that meet the 
inclusion criteria. Therefore, the purpose of this study is to provide information on the Jobe and Docking surgical techniques, the success rates for each technique based on rehabilitation, and outcome measures to evaluate each study using the Coleman Methodology Score (CMS).

\section{METHODS}

Design

The design for this study was be a systemic review of the literature. All studies used were retrieved from searches through specific databases using key words, key phrases, and/or crossreferencing. Each study was evaluated on whether the inclusion/exclusion criterion is met. Of the studies that did meet the inclusion criteria, the Coleman Methodology Score was used to evaluate the quality of the study.

Instrumentation

The CMS consists of 10 criterion that are used to review and evaluate surgical studies. The CMS is based on the CONSORT statement, which is primarily used for randomized control studies. $^{23}$ The CMS was originally created to be used on studies for patellar and Achilles tendinopathy, but is able to be used with any study which involved surgical techniques and rehabilitation. ${ }^{23}$ Each of the 10 criteria is given a score based on the description that criteria match. If a study were to score 100, this would show that the study avoids biases and outside influences. ${ }^{24}$ The quality of the CMS scores that fall between 85 and 100 points was rated as excellent, between 70 and 84 was good, 50 and 69 was fair, and scores that were below 50 points were considered to be poor. $^{25}$

For the criterion that is included in the CMS part 1, each criterion is a single score. Criterion 1 refers to matching the study size to a score listed. Criterion 2 pertains to the number of months for the follow-up. Criterion 3 and 6 refer to the surgical techniques used and how much detail was included in the study. Criterion 4 is scored based on the type of study. Criterion 
5 is scored based on the subjects who were diagnosed with the injury through the use of diagnostic tools. Criterion 7 is scored based upon the detail included in the study on the rehabilitation protocol. For the criterion that is included in the CMS part 2, each criterion is given multiple scores as they pertain to the options listed within each criterion. Criterion 8 is scored based on the how much detail is listed about the outcome of the study. Criterion 9 and 10 is scored based upon if the subjects were recruited and the procedure used to assess the outcomes. See Table C2 for an example of the CMS that was used. Refer to Table C3 for a detailed description of each criterion within the CMS and what each score entails.

Data Sources

First, PubMed (1960-2014), MEDLINE (1960-2014), CINAHL with full text (19602014), SPORTDiscus full text (1960-2014), Science Direct (1960-2014), and Google Scholar (1960-2014) were searched using the terms ulnar collateral ligament, elbow, baseball, reconstruction and treatment. Second, the term ulnar collateral ligament was combined with each of the following terms: surgery, Jobe technique, Docking technique, and rehabilitation. Third, cross-referenced citations were used from studies that included information not found in searches. Studies were found and compared to inclusion and exclusion criteria. Once accepted into the study, the studies were evaluated using the CMS.

Study Selection

Studies were included based on the following criteria: 1) written in English or an English translation; 2) ulnar collateral ligament, baseball athletes, throwing athletes, surgical techniques, elbow, reconstruction, rehabilitation, or various forms of these terms were mentioned in the title of the study; 3) ulnar collateral ligament, baseball athletes, throwing athletes, surgical techniques, elbow, reconstruction, rehabilitation, or various forms of these terms were mentioned in the abstract; 4) ulnar collateral ligament reconstruction and rehabilitation was the main focus 
of the study; 5) the study was an experimental study or cohort study. Studies were excluded on the following criteria: 1) they are not written in or translated into English; 2) studies do not involve the Jobe or Docking technique as a surgical option; and 3) injury did not pertain to the UCL of the elbow.

Data Extraction

All studies that met the inclusion criteria were evaluated using the CMS. Each study included was read first by each evaluator without the CMS. The studies included were read a second time using the CMS. Final score was within a range of 0 to 100 . Once all studies have been read and evaluated, the evaluators' met to compare scores. When discrepancies were found, the evaluators' discussed the criterion in question and agreed on a final score. For this study there were some discrepancies related mostly to clarification. However, after discussion, both evaluators did agree on the final score.

\section{DATA SYNTHESIS}

Study Quality

All twelve studies that met the inclusion criteria were evaluated with the CMS. ${ }^{8,10,13,19-}$ ${ }^{22,26-30}$ A brief summary of the studies and results can be found in Table D1 and D2. The study populations consisted of individuals who were overhead throwing athletes; majority being baseball players, which underwent UCL reconstruction. The studies consisted of the Jobe or Docking surgical techniques. The methodology score ranged from 37 to 62 on the CMS with an average score of 49.2. All scores reported are based on a 100-point scale. The qualities of the studies presented were considered fair ${ }^{10,19,21,22,26,30}$ for six studies and poor ${ }^{8,13,20,27-29}$ for the other six studies. A summary of the scores for each study can be found in Table D3. All individual study scores can be found in Tables D4-15. 
A majority of the studies had a large sample size $(>60)$ reported but failed to address how the patients were enrolled in the study or the percentage used. ${ }^{10,13,21,26,28,30}$ The remaining studies $^{19,20,22,27,29}$ all scored lower on sample sizes for having less than 40 patients; Jobe et al. ${ }^{8}$ scored the lowest with a sample size of 20 patients. These studies all listed a sample size number, but only a few listed a specific inclusion criteria for the study. ${ }^{21,26,30}$ With this lack of information, it is hard to determine if each study's sample size was large enough for the treatment used to be deemed successful or not.

The procedure for description of subject selection process and assessing outcomes on the CMS resulted in a substantial number of points lost per study. Since there was no recruitment rate reported as patient information was taken from the surgeons' files, all studies did not receive 8 points. The remaining studies ${ }^{10,13,28}$ not reporting inclusion criteria lost 5 additional points. The inclusion criteria consisted of history of medial elbow UCL pain, ${ }^{21}$ positive MRI for UCL tear, ${ }^{21}$ UCL insufficiency/injury, ${ }^{21,26}$ over-head throwing athletes, ${ }^{21,30}$ baseball pitchers, ${ }^{26} \mathrm{UCL}$ reconstruction, ${ }^{26,30}$ gracilis autograft used for UCL, ${ }^{26,30}$ and being available for a follow-up ${ }^{26,30}$. Multiple studies ${ }^{10,13,20-22,26,29,30}$ were unable to score any points in the procedure for assessing outcomes (total of 15 points) due to failure to report on how the patients were assessed. Of the studies able to score within this criterion, 4 studies $^{8,19,27,28}$ received 3 points for reporting that a written assessment was used. Two studies ${ }^{27,28}$ scored 3 points for the assessment being completed by subjects and Merolla et al. ${ }^{27}$ was the only study to score points for the investigator being independent of the surgeon.

The scores for the follow-up, number of surgical procedures given, diagnostic tools, procedure descriptions and rehabilitation were considered to be the areas of strength in a majority of the studies. All studies did score the highest possible points ( 5 points) for mean follow-up. All studies reported a follow-up occurring $>24$ months after surgery. ${ }^{8,10,13,19-22,26-30}$ 
The follow-up ranged from 24 months ${ }^{30}$ to 10 years; ${ }^{28}$ with an average of 47 months. This was the only criterion where maximum number of points were achieved for every study. Overall CMS scores were high for descriptions for rehabilitation protocols. Eight studies scored the maximum allotted points (10 points), ${ }^{10,19-22,26,28,29}$ while the remaining 4 studies scored 5 points. ${ }^{8,13,27,30}$

A majority of the studies only reported on one surgical technique used..$^{10,20-22,26,28,29} \mathrm{In}$ three studies, there were two surgical techniques reported with $>90 \%$ of athletes receiving the same technique. ${ }^{13,19,30}$ In one study, it was unclear as to how many athletes underwent the two techniques reported. ${ }^{27}$ Five studies used the Jobe ${ }^{8}$ or modified Jobe technique. ${ }^{10,13,26,28}$ Six studies used the Docking ${ }^{21,22,29,30}$ or modified Docking technique. ${ }^{19,20}$ Of the 12 studies used, 8 studies $^{8,13,19-22,26,29}$ went into considerable detail in describing the technique and all were able to score 5 points for description of surgical procedure. The remaining 4 studies $^{10,27,28,30}$ scored $^{2}$ lower (3 points) for not elaborating on the surgical technique used.

It was noted that the studies, which were published after 2010, scored lower on the descriptions of the surgical technique and elaborated more on the outcomes of the study. In earlier studies, the surgical techniques were explicitly described starting with the original Jobe technique ${ }^{8}$ followed by the modifications to the Jobe technique, ${ }^{10,13,26,28}$ the Docking technique, ${ }^{21,22,27,29,30}$ and modifications of the Docking technique ${ }^{19,20}$. By 2010, the surgical procedures became standard and only modifications or complications that arose were noted. Because of that other studies ${ }^{26-28}$ refer to those earlier published studies. Risk of bias is always a concern in any published surgical study. Yet, there was no definitive conclusion comparing the total CMS score and the year each study was published. Eleven of the 12 studies $^{10,13,19-22,26-30}$ were published after 2000; Jobe et al. ${ }^{8}$ was published in 1986. 
When examining diagnostic tools used to rule in/out UCL injuries, the tools ranged from clinical evaluation to X-ray, MRI, CT scan, ultrasonography and arthroscopy. Not all diagnostic tools provided good sensitivity/specificity for the injury. Between the 12 studies, 8 studies reported positive results for the use of MRI, ultrasound, x-ray or another form of diagnostic tools. ${ }^{10,19,21,22,26,27,29,30}$ This limitation of positive diagnostic tests leads to a conclusion that surgeons relied on diagnostic arthroscopy to view the UCL and make a final diagnosis.

The areas that seemed to have a negative impact on the overall score for each study were the type of study and outcomes, in addition to assessing the outcomes and the subject selection (which were described previously). For the type of study, all studies were retrospective cohort studies or case series, which affected each study as none received a score above $0 .{ }^{8,10,13,19-22,26-30}$ For the outcome criterion and assessing outcomes, only one study ${ }^{27}$ scored a 10 out of 10 for points possible. Three studies ${ }^{10,13,29}$ missed 2 points in outcome criteria due to the outcome measures not being clearly defined. This occurred because the studies did not elaborate on the overall outcomes or summarized the outcomes in a limited number of paragraphs with little detail provided. Eleven studies scored lower for not reporting good sensitivity with the use of outcomes. ${ }^{8,10,13,19-22,26,28-30}$ The only studies that scored above a 4 on the outcomes was based on good reliability being reported (through the Conway-Jobe Scale/Conway Scale). ${ }^{19-22,26,28,30}$ Information regarding the studies individual CMS can be found in Tables D3-15. Jobe Technique

There were seven studies that investigated the Jobe technique. ${ }^{8,10,13,26-28}$ Jobe was the creator of the surgical technique, ${ }^{8}$ but his study scored the lowest on the CMS 37/100 (Table D9). The quality of the study was reported to be poor. The average return to play (RTP) rate for the 16 throwing athletes was $67 \% .^{8}$ 
In conclusion, the original Jobe technique was deemed to be the least successful technique used. There are multiple factors that could have lead to this with the main factor relating to the time the surgery was performed as this was the first surgical technique used. As modifications were explored, the success rates began to increase. The time era in which the surgery was performed could have also been another factor. Jobe et al. ${ }^{8}$ reported the success rates in 1986. The next study that evaluated the Jobe technique was published in $2000 .{ }^{13}$ Modified Jobe Technique

Azar et al., ${ }^{13}$ Cain et al., ${ }^{10}$ Dugas et al., ${ }^{26}$ and Osbahr et al., ${ }^{28}$ all used a modified Jobe technique with two specific modifications. These modifications were an ulnar nerve transposition and not detaching the flexor pronator mass from the medial epicondyle. ${ }^{10,13,26,28}$

Azar et al. ${ }^{13}$ investigated the surgical records of 78 males who underwent UCL reconstruction and 13 males who underwent UCL repair. With the modifications used, there were fewer complications reported for ulnar nerve pathologies. There was a 79\% RTP reported. The study scored the second lowest for the CMS with a score of 39/100, which indicated the quality of the study to be poor (Table D4).

Cain et al. ${ }^{10}$ investigated the surgical records for $1266 \mathrm{UCL}$ reconstructions and $15 \mathrm{UCL}$ repairs. There were 1253 males in the study and 28 females. There was an $83 \%$ RTP rate to previous level of competition or higher. The CMS for this study was 50/100, which indicated the quality of the study to be fair (Table D6).

Dugas et al. ${ }^{26}$ investigated the surgical records for 120 patients who underwent UCL reconstruction. The patients were divided into two groups for the study (bony and nonbony). The bony group consisted of patients who had intraligamentous bony abnormalities present. The nonbony group was those who did not have a palmaris longus. Forty-two patients were placed in the bony group with an outcome of $81 \%$ RTP rate. Seventy-eight patients were placed in the 
nonbony group with an outcome of $89 \%$ RTP rate. The study used the Conway-Jobe scale to determine the success rate for the surgery and the nonbony group presented with 71 of the 78 patients having an excellent rating for RTP. There were only 34 of the 42 patients in the bony group who reported excellent rates. The excellent rating refers to athletes who were able to return to previous level of competition or higher. The CMS for this study was $62 / 100$, which indicated the quality of the study to be fair (Table D8).

Osbahr et al. ${ }^{28}$ investigated the surgical records for 313 patients who underwent UCL reconstruction. Only 256 of the 313 patients were available for follow up at 10 years. It was reported that there was a $93 \%$ satisfaction rate for the surgical patients. After a mean follow-up of 10 years, $95 \%$ of the athletes reported retired from the sport, with reasons not related to the elbow. Only $5 \%$ of the patients in the study remained active in sport. It was noted that few reported pain after surgery. The RTP rate was stated to be $83 \%$. The CMS for this study was $47 / 100$, which indicated the quality of the study to be poor (Table D12).

In conclusion, the modified Jobe technique reported better outcomes in terms of RTP percentages over the original technique reported by Jobe. The RTP rate ranged between $79 \%{ }^{13}$ and $83 \%,{ }^{10,28}$ with an average of $81.5 \%$. Despite the higher RTP rates, the range for the CMS scores were $39-62$, with two studies ${ }^{13,28}$ considered to be poor and two studies ${ }^{10,26}$ considered to be fair. Based on the CMS scores, it is inconclusive if the modified Jobe technique is a successful surgical procedure.

Docking Technique

There were seven studies included that investigated the Docking technique. ${ }^{19-22,27,29,30}$ Rohrbough et al. ${ }^{22}$ was deemed to be the creator of the Docking technique. The methods used for this technique were similar to the Jobe technique, with some modifications made, which appeared to lead to more successful reported outcomes. A description of the technique and 
modifications can be found in Table B2. The study investigated the surgical records for 36 patients, 35 males and 1 female. A 92\% RTP rate was reported with 21 of the 36 patients returning to their previous level of competition. Twelve of the 36 patients reported being able to advance to higher levels of competition after surgery. The CMS for this study was $51 / 100$, which indicated the quality of the study to be fair (Table D14).

Dodson et al. ${ }^{21}$ investigated the surgical records for 100 male over-head throwing athletes. There was an overall RTP rate of $97 \%$. The RTP rate reported for those returning to the previous level of competition or higher was $90 \%$, while $7 \%$ of athletes moved down to a lower level of competition. The CMS for this study was $62 / 100$, which indicated the quality of the study to be fair (Table D7).

Paletta et al. ${ }^{29}$ investigated the surgical records for 25 baseball players. The study concluded with 23 players (92\%) returning to play at the same level of competition or higher. The CMS for this study was $46 / 100$, which indicated the quality of the study to be poor (Table D13).

Savoie et al. ${ }^{30}$ investigated the surgical records for 123 overhead-throwing athletes. While only 116 were used in the study, 6 players were reported to not RTP. It was reported that there was a RTP rate of $88 \%$, with 33 players returning to higher levels of competition and 64 returning to a previous level of competition. On the Conway-Jobe scale, 93 players were classified as excellent for RTP and 15 players were classified as good. Allografts were used in all surgeries and it was reported that this had an advantage as only one surgery was performed, rather than two to remove the graft. The CMS for this study was 52/100, which indicated that quality of the study to be fair (Table D15).

In conclusion, the Docking technique is noted to be a successful surgical technique used for UCL reconstruction based on percentages reported for success rate within the studies. The 
success rate ranged from $88 \%{ }^{30}$ to $97 \%,{ }^{21}$ with an average of $92.25 \%$. However, with the CMS scores reported to be fair and poor, it is inconclusive if the Docking technique is considered to be a successful treatment option for UCL reconstruction.

Modified Docking Technique

Only two studies mentioned modifications with the Docking technique. ${ }^{19,20}$ These modifications pertained more to the tendon graft used than modifying the procedure. Bowers et al. ${ }^{20}$ described what to do with the excessive graft end. That part was overlapped with the graft used and sewed down. Koh et al. ${ }^{19}$ reported using a 3-strand technique for the tendon graft half way through the study. The first 12 patients had a 2-strand technique graft used, while the remaining 8 patients received the 3 -strand technique. No outcomes were reported comparing the success rate of those who received the 3 -strand over the 2 -strand.

Bowers et al. ${ }^{20}$ investigated the surgical records for 21 over-head throwing athletes. It was reported there was a 90\% RTP rate with 19 athletes returning to play within 1 year to a previous level of competition (excellent classification on the Conway-Jobe scale). Only 2 athletes moved down to a lower level of competition (good classification on the Conway-Jobe scale). The CMS for this study was $46 / 100$, which indicated the quality of the study to be poor (Table D5).

Koh et al. ${ }^{19}$ investigated the surgical records for 20 high-level baseball players. Eighteen athletes RTP to the same level of competition or higher, while only 2 players reported returning to play but not to pitching (remained in baseball but switched positions). On the Conway-Jobe scale, 17 players were classified as excellent for RTP, while 2 players were classified as good. With 19 of the 20 athletes returning to play, the success rate can be reported as being $95 \%$ RTP rate. The study concluded the Docking technique was a reliable technique to be used for UCL 
reconstruction for a successful RTP. The CMS for this study was 51/100, which indicated the quality of the study to be fair (Table D10).

In conclusion, with the RTP rate was similar to that of the Docking technique, it could be noted that both the modified Docking and Docking technique are viable treatment options for a successful RTP based on reported percentages of success rates within the studies. With an average RTP rate of $91 \%$ for the two studies using a modified Docking technique, this average is only $1 \%$ below the average RTP for the Docking technique. However, based on CMS scores, the quality of the studies that used the modified Docking technique were fair to poor. This could lead to the surgical technique's success rate to be deemed inconclusive.

Combined Jobe and Docking Techniques

Merolla et al. ${ }^{27}$ investigated the surgical records for 26 patients who underwent UCL reconstruction. Only 15 were used in the study. Of these 15 patients, only 5 patients underwent UCL reconstruction with the use of the Jobe technique, while 8 used the Docking technique. The two groups did not report the results individually. On the Conway-Jobe scale, 10 patients were classified as excellent for RTP. The greatest complication noted for the surgeries was the widening of the tunnels to accommodate the size of the graft. By widening the tunnels, this would lead to weakening the bone. Because of this, the grafts had to be thinned. Only 6 athletes were reported to have a RTP rate to the previous level of competition or higher. The CMS for this study was $47 / 100$, which indicated the quality of the study to be poor (Table D11). Rehabilitation Protocol

Descriptions for rehabilitation protocols were very brief in four studies ${ }^{8,20,21,29}$ and only included a short paragraph. These studies only mentioned certain week progressions related to discontinuation of the brace, start/progressions of exercises or when full ROM should be restored (6 weeks), and when sport specific exercises can begin (at the 9 week mark). There were five 
studies, ${ }^{10,13,19,22,30}$ which mentioned more specific week progressions, with one of these studies ${ }^{13}$ considered the most detailed.

There were three studies that did not report a rehabilitation protocol at all. ${ }^{26-28}$ Only two studies ${ }^{26,28}$ lead the reader to a rehabilitation focused article published by Wilk et. al. ${ }^{31}$ Merolla et. al. ${ }^{27}$ had no mention of a rehabilitation protocol, only that the brace was discontinued 30 days post-op, at 60 days post-op resistance exercises were initiated and 90 days post-op was when sport specific exercises could begin. ${ }^{27}$

For this section, scores were based on return rates and not rehabilitation descriptions. When strictly looking at return to play rates or success rates, all studies should receive the highest number of points. However, when looking at rehabilitation protocol descriptions, a majority of the studies should have a different score reported. Two of the studies ${ }^{13,27}$ that scored a 5 on the CMS varied on an adequate rehabilitation protocol description. Azar et al. ${ }^{13}$ was the one study that went into the most detail for the rehabilitation protocol followed by Cain et al., ${ }^{10}$ and Koh et al., ${ }^{19}$ for weekly progressions (refer to Table B3 for rehabilitation protocol overviews). Merolla et al. ${ }^{27}$ did not report on the rehabilitation protocol at all, as that study was mostly concerned with outcomes. Due to a success rate between $60-80 \%$, the study scored a 5 . In conclusion, rehabilitation protocols cannot be determined to have variation between them based on information provided within the studies. Some studies ${ }^{26,28}$ referred to a study commonly used for UCL rehabilitation, while others mentioned only the physicians protocol..$^{8,10,13,19-22,27,29,30}$ Based on the brevity of what was reported and in combination with one detailed protocol a weekly rehabilitation protocol could be established. However, the final definitive factor is the surgeon's preference in regard to a progression following UCL reconstruction. 


\section{DISCUSSION}

The purpose of this study was to examine and analyze the current literature on the Jobe and Docking surgical techniques for UCL reconstruction and the rehabilitation that follows; specifically, to review the two surgical techniques in relation to success and return to play rates. The six research questions that were proposed were related to surgical techniques and rehabilitation. The surgical questions included what surgical techniques (Jobe, Docking, or newer variations) are successful, and if there is a preference on the surgical technique used between different levels of competition in UCL reconstruction. The rehabilitation questions were in relation to a difference in rehabilitation, exercises progressions and throwing progressions between the surgical techniques or those who undergo the conservative approach, and if there is a difference in the return to play rates. Based on the information provided in the studies and the CMS scores, very few questions were answered.

To elaborate further on the research questions, this systematic review had six experimental hypotheses. A detailed list of hypotheses is found in Appendix A. The sixth hypothesis was the only one to be confirmed. The hypothesis was that there would be enough information in studies to be individually scored on the Coleman Methodology Score. The first (the Docking technique will be found to be more successful for surgery and RTP rates) was partially confirmed. As preference for surgical techniques at each level will be based on experience of the attending surgeon, the studies that reported using the Docking and modified Docking technique had higher percentages of RTP rates over the Jobe and modified Jobe techniques. However, based on the CMS scores, the decision on what technique was deemed to be successful was inconclusive. Of the studies that elaborated on rehabilitation protocols, there was little mention of a biomechanical evaluation. For these hypotheses to be confirmed, more 
information should be provided in the studies in relation to subject selection and outcomes assessment.

The remaining second (rehabilitation between different surgical techniques will be slightly similar, with differences found in earlier progressions and more conservative approaches), third (throwing progressions will be found to begin at different time points depending on the surgical technique used), fourth (a change in throwing biomechanics will provide for a decreased rate of re-injury) and fifth (those who undergo surgery will have a more successful RTP rate and will return to pre-injury levels over those who take on the non-surgical options) hypotheses were unconfirmed. There were no differences noted between different surgical techniques used for rehabilitation, and in studies where the rehabilitation protocol was reported, the throwing progressions all began around the same time. None of the studies evaluated reported the athletes undergoing a conservative approach before surgery.

Coleman Methodology Scores ranged from 37 to 62 points with an average score of 49.2. Overall, the CMS scores were considered to be fair or poor. Individually, the strength of the CMS scores were follow-up mean times, number of surgical procedures, description of the surgical technique, and reported compliance rates of rehabilitation. All studies reported followups occurring $<24$ months post-operative; which led to the maximum number of points scored for each study. A majority of the studies reported on only one surgical technique being utilized and the surgical procedures were adequately described. For rehabilitation, over half of the studies who reported success rates of $>80 \%$ scored the most points. The successful rehabilitation rates were based on the compliance of the athletes.

The diagnostic certainty of the patients having the injury prior to surgery was considered to be a strength and a weakness. A total of 8 studies scored the maximum number of points allotted. However, since this is only $66 \%$ of the studies, it cannot be concluded whether this is a 
strength or true weakness. Studies reported that all patients had a confirmed UCL tear, but some studies reported on how the injuries did not show up with the use of diagnostic tools until a diagnostic arthroscopy was performed.

The major limitations within the CMS scores occurred within the criterions pertaining to the type of study, subject selection process, outcome sensitivity, and assessing the outcomes. All the involved studies were either retrospective cohort studies ${ }^{26}$ or case series. ${ }^{8,10,13,19-22,27-30}$ Due to the type of studies used, no points could be awarded to any study. It is questioned whether a randomized control study can be conducted with surgical studies. Randomization of patients could be considered as unethical especially in deciding which patient would receive one of two surgical techniques for the purpose of research. Therefore, prospective cohort, retrospective cohort or case series appear to be the design of published surgical studies. With the studies following the aforementioned designs, information on subject selection criteria was not reported and no study scored points for this criterion. All 12 studies recruited the patients from surgeons' files. This allows for a selection bias because it of the decreased chance of randomization to occur within the study. In all the studies used, no patients were randomly allocated or recruited.

The authors of the studies failed to report on whether the outcomes instrument used had the appropriate sensitivity, and most studies did not clearly define how the outcomes were measured. Only one study ${ }^{27}$ reported good sensitivity for the outcomes measures used. The Conway-Jobe scale was used by 8 studies ${ }^{19-22,26-28,30}$ to report RTP outcomes. The other studies $^{8,10,13,29}$ reported percentages on athletes who returned to previous level of competition or higher, but did not specifically report outcomes on the Conway-Jobe scale. Based on the RTP percentages provided $\left(88 \%-97 \%{ }^{19-22,27,29,30}\right)$, it would appear that one surgical technique might be better than the other. For example, the range for RTP percentages for the Docking technique (88\%-97\% $\left.{ }^{19-22,27,29,30}\right)$, were considered good. However, the CMS scores were low. When 
reported CMS scores are low, various types of bias could be apparent and lead to threats to internal and external validity. With limitations evident in surgical studies, including low CMS scores, it cannot be determined whether one specific surgical technique is the best treatment option. Further, from the information provided, it could not be determined whether the patient assessment was a self-report obtained by the surgeon or completed by the patient. The studies mentioned phone interviews, but it was not specified if these interviews were primarily a written assessment or completed by the patient themselves. With phone interviews, the interviewer can sway how a patient responds to a question based on the tone of voice of the interviewer, how the question was asked and if there was any assistance with answering the questions. ${ }^{24}$ Jobe Technique

With this technique as the first surgical reconstruction technique in $1974,{ }^{8}$ it was considered to be better than UCL repair. It provided baseball players with an opportunity to continue a baseball career that previously was considered over. However, the first surgical technique experienced multiple complications that were not determined immediately. Some of the complications were the transposition of the ulnar nerve and the incising of the flexor pronator muscle group to reach the torn UCL. Further, based on the year of publication, evaluation material in the Coleman Methodology Score may not have been required. Although this type of surgery was considered the "gold standard of surgery", several threats to internal and external validity were not considered, and may have lead to a low score on the CMS.

Modified Jobe Technique

With the modifications to the original technique, the success rate for the four studies $^{10,13,26,28}$ that reported these modifications were good. The modifications were to not perform an ulnar nerve transposition, unless deemed necessary, and to avoid detachment of the flexor-pronator mass. Modifications, especially avoiding ulnar nerve transpositions, decreased 
temporary and or permanent nerve damage in comparison to the original Jobe technique. A limitation to these studies was especially noted in outcomes. Only one study, Dugas et al., ${ }^{26}$ reported using the Conway-Jobe scale. Also, outcomes were self-reported on return to activity one year post-operatively. Rarely did outcomes report on ROM or strength. Despite having better outcomes rates reported the CMS outcomes were low. Overall CMS scores on the four studies were fair to poor.

Docking Technique

The technique was introduced as a variation from the Jobe technique in regard to the anatomical positioning of the graft and the tunnel placement. The concept was that the graft would have a greater anatomical positioning using the "Y-shape" with tunnels drilled to create a pathway similar to the alignment prior to rupture. With these changes, the new "ligament" has a better anatomical advantage over the Jobe technique; which could lead to biomechanical advantages as well. Despite the success, it was noted that a weakness of this technique could be due to the number of tunnels drilled (4 total). ${ }^{22}$ A strength of Savoie et al. ${ }^{30}$ was the use of an allograft. By using an allograft, only one surgical procedure is performed. This would help with return to play rates by avoiding complications noted with the use of ispilateral or contralateral grafts. Despite this, the studies that reported using the Docking technique had CMS scores considered fair to poor.

Modified Docking Technique

With the modifications to the original technique, the success rates with the two studies that used a modified technique were good. The modifications were switching from a 2-strand approach to a 3 -strand approach ${ }^{19}$ and sewing the excessive graft end. ${ }^{20}$

The strengths of Koh et al. ${ }^{20}$ was the study introduced a new modification of changing a 2-strand technique with the graft to a 3-strand technique. This was hypothesized to increase the 
strength of the graft used, however, no outcomes were reported comparing the 2-strand to the 3strand technique. A strength for Bowers et al. ${ }^{19}$ was the study focused on the overall kinetic chain for rehabilitation instead of only the elbow. Similar to the Jobe and modified Jobe technique, the Docking and modified Docking techniques all reported higher outcomes percentages per study. There were fewer limitations noted with the two studies ${ }^{19,20}$ that reported using a modified Docking technique. Both studies reported the modifications and outcome rates. However, based on low CMS outcomes reported to be fair to poor, it remains inconclusive which surgical technique should be advocated to use for a UCL reconstruction.

Combined Jobe and Docking Technique

Only one study ${ }^{27}$ reported outcomes for using both the Jobe and Docking techniques. There were limitations with the study by not reporting outcomes comparing the two techniques. The authors reported how many patients received what technique, but the outcomes were reported as a whole. Another limitation to this study was the authors reported that techniques lead to a complication of widening the bone tunnels to accommodate the graft. The surgeons did not like the idea to increase the diameters of the tunnels, as this could lead to weakening of the bone. Thus, the graft was thinned to accommodate the tunnel diameter. There were no outcomes reported on if thinner grafts lead to a decrease in outcomes.

Rehabilitation Protocol

Comparing the CMS scores for the description of the postoperative rehabilitation, a majority of the studies ${ }^{10,19-22,26,28,29}$ scored high on this criterion. However, a major limitation was the wording of the criterion. A score was provided if the rehabilitation protocol was well described with $>80 \%, 60 \%-80 \%$ of the patients returning, protocol was not reported, or $<60-80 \%$ of the patients returning. When scoring the studies, it was found that a study could have higher outcomes reported but did not do an adequate job in describing the rehabilitation protocol 
followed. This leads to problems on what the score really should be based on; the descriptions of the rehabilitation protocol or the outcomes reported. It was difficult to decide what an appropriate score would be for studies who did not report a rehabilitation protocol but did report outcomes $>80 \%$. It could be suggested to modify the CMS criterion into two separate scoring questions. One score would be for reported RTP outcomes while another score would be for the description of the rehabilitation protocol.

Overall, it can be reported that there were little to no differences reported for rehabilitation protocols from study to study. The differences that were noted were related to major milestones. When the brace was discontinued varied between 2 weeks, ${ }^{8,31} 6$ weeks, ${ }^{20,21,29}$ and 8 weeks. ${ }^{13}$ Savoie et al. ${ }^{30}$ utilized the brace through the whole rehabilitation process and the beginning of the throwing progression before the brace was discontinued at 4 and half months. Full ROM was to be achieved at 6 weeks. ${ }^{10,13,19,20,22,29,30,31}$ Throwing progressions were started at 12 weeks, ${ }^{30} 16$ weeks, ${ }^{10,20,21,22,29,31}$ or 18 weeks ${ }^{19}$. These differences did not deviate much between the studies. It was noted that two of the studies ${ }^{26,28}$ only reported on the rehabilitation protocol published by Wilk et al.. ${ }^{31}$ This study appeared to be the "gold standard" for UCL reconstruction. The Wilk et al. ${ }^{31}$ rehabilitation protocol follows similar milestones reported in other studies. The study went into a week-to-week progression, along with dividing the phases for exercises that should be incorporated and when to progress the exercises. ${ }^{31}$ Limitations of the Study

There were a total of 12 studies that met the inclusion criteria for this study. Overall the methodology quality scores were between fair to poor. There were multiple areas in which the studies were considered to be fair to poor. All of the studies were considered to be retrospective cohort or case series. With surgical techniques and outcomes, it could be questioned as ethical to conduct a randomized control study by using different surgical techniques. It would not be 
deemed fair to give some individuals one surgical technique over another just for the purpose of research, especially in using baseball athletes as subjects. Cadaver studies are used to evaluate surgical techniques prior to use in the clinical setting. However, a cadaver study will not report on return to throwing, but rather on anatomical and biomechanical loading. Another concern is that if studies were published after the techniques were well developed, the only explanations reported would be the deviations from the technique with modifications or complications.

In regard to the rehabilitation protocols, or the lack of protocols reported, it was difficult to detect the variations between surgical techniques. None of the studies noted any modifications made to make the protocol more individualized. It cannot be assumed that all athletes were on the same timeline of milestones without any set backs or complications. Further, UCL reconstruction surgeries should elaborate on rehabilitation progressions reported.

For the outcomes reported, there were some limitations. The only reliable tool reported within the majority of the studies used to assess the outcome rates was the Conway-Jobe scale. This scale was found to be reliable, but is subjective and based on whether the athlete returned to a previous level or higher, went down a level of competition, or never returned. There were little to no reports of any sensitivity reported either. With no measureable outcomes reported, it is difficult to report on the benefits of a surgical technique. Definitely, studies reporting on more objective outcomes are needed.

\section{Clinical Relevance}

The main purpose for this study was to evaluate UCL reconstruction and to provide athletic trainers with that information. This injury is not uncommon in the sport of baseball, but the type of surgical techniques may not be known. Although only two surgical techniques and their variations were explored within this study, there is still a fair amount of information that may not be known. 
Based on the CMS scores, one surgical technique or several surgical techniques are not any better than the other. Although, the ultimate decision on which surgical technique to use is based on the attending surgeons preference, this study was intended to provide information on these two techniques to assist baseball athletic trainers with an athlete scheduled for UCL reconstruction. What is known is that an athletic trainer can be provided with a timeline for return to activity following a lengthy rehabilitation protocol. However, as surgeons may have a preferences on how to approach the rehabilitation aspect of this injury, and with limited information on rehabilitation reported in studies, the athletic trainer may need to work closely with the physician in regard to progression on return to throwing and activity.

\section{CONCLUSION}

With CMS scores being fair to poor, no recommendation can be made on what surgical technique can be considered the best option for UCL reconstruction. Further, the outcomes reported within each study were based on return to sport or not and whether if the return was up or down a level. With the CMS, there were many areas of strengths and other areas that lead to great weakness. The areas that provided strength to the study were sample sizes, follow-ups, number of surgical procedures, descriptions of the techniques, reported compliance rates of rehabilitation and subject selection. The weaknesses that were found within the CMS scores were the type of study, outcome sensitivity, subject selection process and assessing the outcomes. Due to weaknesses, studies did not score a substantial number of points in the criteria provided on the CMS. Further research is necessary to determine surgical success. However, with a surgical technique based on surgeon's preference and experience with limited objective outcomes, results may remain inconclusive. 


\section{REFERENCES}

1. Dick R, Sauers El, Agel J, Keuter G, Marshall SW, McCarty K, McFarland E. Descriptive epidemiology of collegiate men's baseball injuries: national collegiate athletic association injury surveillance system, 1988-1989 though 2003-2004. J Athl Train. 2007;42(2):183-193.

2. Posner M, Cameron KL, Wolf JM, Belmont Jr PJ, Owens BD. Epidemiology of major league baseball injuries. Am J Sports Med. 2011;39(8): 1676-1680.

3. Meyers A, Palmer B, Baratz ME. Ulnar collateral ligament reconstruction. Hand Clin. 2008;24:53-67.

4. Patel RM, Lynch TS, Amin NH, Calabrese G, Gryzlo SM, Schickendantz MS. The thrower's elbow. Orthop Clin N Am. 2014;45:355-376.

5. Reinold MM, Fleisig GS, Andrews JR. Biomechanics and rehabilitation of elbow injuries during throwing. Athletic Ther Today. 2000;5(3):12-18.

6. Safran M, Ahmad CS, Elattrache NS. Ulnar collateral ligament of the elbow. Arthroscopy. 2005;21(11):1381-1395.

7. Brabston EW, Genuario JW, Bell JE. Anatomy and physical examination of the elbow. Oper Tech Orthop.2009;19:190-198.

8. Jobe FW, Stark H, Lombardo SJ. Reconstruction of the ulnar collateral ligament in athletes. J Bone Joint Surg Am. 1986;68(8):1158-1163.

9. Conway JF, Jobe FW, Glousman RE, Pink M. Medial instability of the elbow in throwing athletes. J Bone Joint Surg Am. 1992;74-A(1):67-83.

10. Cain EL, Andrews JR, Dugas JR, Wilk KE, McMichael CS, Walter JC, Riley RS, Arthur ST. Outcome of ulnar collateral ligament reconstruction of the elbow in 1281 athletes: results in 743 athletes with minimum 2-year follow up. Am J Sport Med. 2010;38(12):2426-2434.

11. Jiang JJ, Leland Jm. Analysis of pitching velocity in major league baseball players before and after ulnar collateral ligament reconstruction. Am J Sport Med. 2014;42(4):880-885.

12. Anz AW, Bushnell BD, Griffin LP, Noonan TJ, Torry MR, Hawkins RJ. Correlation of torque and elbow injury in professional baseball pitchers. Am J Sport Med. 2010;38(7):1368-1374.

13. Azar Fm, Andrews JR, Wilk KE, Groh D. Operative treatment of ulnar collateral ligament injuries of elbow in athletes. Am J Sport Med. 2000;28(1):16-23. 
14. Bennett JM, Mehlhoff TL. Reconstruction of medial collateral ligament of the elbow. $J$ Hand Surg. 2009;34A:1729-1733

15. Dines JS, Frank JB, Akerman M. Glenoumeral internal rotation deficits in baseball players with ulnar collateral ligament insufficiency. Am J Sport Med. 2009;37(3):566570 .

16. Hurd WJ, Kaufman KR. Glenohumeral rotational motion and strength and baseball pitching biomechanics. $J$ Athl Train. 2012;47(3):247-256.

17. Lyman S, Fleisig GS, Waterbor JW, Funkhoser EM, Pulley L, Andrews JR, Osinski ED, Roseman JM. Longitudinal study of elbow and shoulder pain in youth baseball pitchers. Med Sci Sports Exerc. 2001;33(11):1803-1810.

18. Jones KJ, Dines JS, Rebolledo BJ, Weeks KD, Williams RJ, Dines DM, Altchek DW. Operative management of ulnar collateral ligament insufficiency in adolescent athletes. Am J Sport Med. 2013;42(1):117-121.

19. Koh JL, Schafer MF, Keuter G, Hsu JE. Ulnar collateral ligament reconstruction in elite throwing athletes. Arthroscopy. 2006;22(11):1187-1191.

20. Bowers AL, Dines JS, Dines DM, Altchek DW. Elbow medial ulnar collateral ligament reconstruction: Clinical relevance and the docking technique. J Shoulder Elbow Surg. 2010;19:110-117.

21. Dodson CC, Thomas A, Dines JS, Nho SJ, Williams RJ, Altchek DW. Medial unlar collateral ligament reconstruction of the elbow in throwing athletes. Am J Sport Med. 2006;34(12):1926-1932.

22. Rohrbough JT, Altchek DW, Hyman J, Williams RJ, Botts JD. Medial collateral ligament reconstruction of the elbow using the docking technique. Am J Sport Med. 2002;30(4):541-548.

23. Jakobsen RB, Engebretsen L, Slauterbeck JR. An analysis of the quality of cartilage repair studies. J Bone Joint Surg Am. 2005;87(10):2232-2239.

24. Coleman BD, Khan KM, Maffulli N, Cook JL, Wark JD. Studies of surgical outcome after patellar tendinopahy: clinical significance of methodological deficiencies and guidelings for future studies. Scand J Med Sci Sports. 2000;10:2-11.

25. Longo UG, Loppini M, Petrillo S, Berton A, Maffulli N, Denaro V. Management of cervical fractures in ankylosing spondylitis: anterior, posterior, or combined approach? British Medical Bulletin. 2015;0(0):1-10

26. Dugas JR, Bilotta J, Watts CD, Crum JA, Fleisig GS, McMichael CS, Cain EL, Andrews JR. Ulnar collateral ligament reconstruction with gracilis tendon in athletes with intraligmentous bony excision. Am J Sport Med. 2012;40(7):1578-1582. 
27. Merolla G, Del Sordo S, Paladini P, Porcellini G. Elbow ulnar collateral ligament reconstruction: clinical, radiographic, and ultrasound outcomes at a mean 3-year followup. Musculoskelet Surg. 2014;98(Supp1 1):S87-S93.

28. Osbahr DC, Cain EL, Raines T, Fortenbaugh D, Dugas JR, Andrews JR. Long-term outcomes after ulnar collateral ligament reconstruction in competitive baseball players. Am J Sport Med. 2014;42(6):1333-1342.

29. Paletta GA, Wright RW. The modified docking procedure for elbow ulnar collateral ligament reconstruction; 2-year follow-up in elite throwers. Am J Sport Med. 2006;34(10):1594-1598.

30. Savoie FH, Morgan C, Yaste J, Hurt J, Field L. Medial ulnar collateral ligament reconstruction using hamstring allograft in overhead throwing athletes. J Bone Joint Surg Am. 2013;95:1062-1066.

31. Wilk KE, Arrigo CA, Andrews JR, Azar FM. Rehabilitation following elbow surgery in the throwing athlete. Oper Tech Sports Med. 1996;4(2):114-132 
APPENDICES 


\section{APPENDIX A}

\section{Research Question}

\section{THE PROBLEM}

In the sport of baseball, injuries from sprains and strains to various muscles and ligaments are evident. About $45 \%$ of baseball injuries involve the upper extremity. ${ }^{1}$ Injuries to the glenohumeral joint and the elbow predominate and the players most affected by these injuries are pitchers, accounting for the highest rates of injuries. ${ }^{1,2}$ Although elbow injuries are not as common as glenohumeral joint injuries, ulnar collateral ligament (UCL) injuries can be the most debilitating. ${ }^{2}$ One study conducted from 2002-2008 on injuries to Major League Baseball players found that an average of $26.3 \%$ of pitchers sustained elbow injuries each season, which is the second highest next to the glenohumeral joint $(30.7 \%) .{ }^{2}$ Aside from pitchers having the second highest injury rate for elbow injuries, starting pitchers have been found to have a significantly greater chance of UCL injury over other pitchers. ${ }^{32}$ One author has reported that a baseball player is 3 times more likely to sustain an injury to the elbow during a game over practice. ${ }^{1}$ This could be due to the risky behavior some pitchers have of continuing to pitch through pain and throwing pitches harder/faster during games over practice. ${ }^{1}$

Injuries to the UCL are the most commonly seen in overhead throwing athletes. The UCL is a ligament that is composed of three bands, with the primary stabilizer and most commonly affected being the anterior collateral band. The nature of the injury to the UCL leading to failure of the ligament and causing a tear is classified as a more chronic type injury. However, the UCL does not just automatically tear without there being little mircotears occurring over time. Once these tears become more extensive, forces placed on the elbow exceed what the medial aspect of the elbow can handle, and the UCL is torn. ${ }^{3-12}$ 
There are numerous intrinsic and extrinsic risk factors that contribute to a UCL tear and the potential for surgery. Aside from mircotears over time causing the chronic nature of the injury, $5,6,8-10,12,14,15$ the mechanics of the initial injury, biomechanical abnormalities at the glenohumeral joint and elbow ${ }^{12,15,16}$ and throwing errors ${ }^{17}$ all commonly lead to the failure of the UCL. Determining why the UCL failed and the surgical techniques chosen can help in the understanding the extensive rehabilitation process and the likeliness of the athlete returning to throwing.

The microtears in the UCL are caused over time and are due to an excessive valgus moment, which occurs at the elbow causing stress on the UCL. ${ }^{9}$ When a UCL injury occurs, there are two methods of treatment for the injury. ${ }^{8}$ One is to correct the biomechanics ${ }^{13,15,16}$ with a more conservative approach of rehabilitation. ${ }^{8-10}$ The second approach is to surgically correct the UCL tear with an extensive rehabilitation that includes correcting the throwing biomechanics. ${ }^{13,16}$ Most physicians will prefer a more conservative approach in treating the injury to prevent unnecessary surgeries and an extensive rehabilitation process. ${ }^{3,4,8-10}$ But according to Jobe ${ }^{8}$ surgery is the best method to treat UCL injuries.

In the past, UCL injuries ended an athlete's career in baseball. But since Dr. Frank Jobe developed and performed a successful reconstruction of the UCL in 1974, this injury is no longer deemed career ending. ${ }^{8}$ Since surgery techniques have evolved the return to play rate has increased over the years. ${ }^{8-10}$ With increasing number of pitchers returning and the evolution of surgical technique, one questions why this injury is occurring and whether one technique is more successful than another.

There are an increasing number of variations of surgical techniques used to reconstruct a UCL tear. ${ }^{8,9,18}$ All of the techniques are variations from the first technique. This technique is called the Jobe technique. Another popular technique is the Docking technique. It is a slight 
variation from the Jobe technique but is found to be more successful. ${ }^{18,19}$ Aside from these two, there are other techniques surgeons use based on success rates and personal preferences. ${ }^{8,21}$

The surgical procedure for the Jobe technique primarily uses an autograft tendon of the palmaris longus from the contralateral wrist. ${ }^{8-10,19}$ There are typically 3 bands used of the tendon graft for this procedure. ${ }^{33}$ The ulnar nerve typically is transposed, to protect the nerve during surgery and tunnels are drilled though the humerus and ulna. ${ }^{8,9}$ The autograft tendon is passed through these tunnels and connected together, forming a "figure- 8 " pattern. ${ }^{8,9}$ The other variation of this technique is the Docking technique. This technique is similar to the Jobe technique but instead of using 3 bands, the Docking technique will use 4 bands. ${ }^{33}$ The surgery for this technique consists of drilling 3 tunnels and the tendon graft is docked into place forming a "Y" figure, as opposed to the "figure- 8 ". 19

The contraindication to each surgical technique can be the extensive rehabilitation process. As each procedure is preformed with variations from the other techniques, the rehabilitation processes are slightly different as well. This slight variation could increase rehabilitation time or decrease time out. The success rate for the different surgeries is also another key factor in the rehabilitation process. Rushing an athlete back too soon could lead to another failed UCL or prolong the process of return to throwing, pitching or the sport in general.

With all rehabilitation protocols there are specific goals that must be met throughout the process and also for return to play. Some of these goals for returning the athlete to play are a full, pain free range of motion and strength at both the elbow and glenohumeral joints. ${ }^{8}$ The athlete should also be able to maintain adequate balance while throwing, rhythm and coordination while throwing. ${ }^{8}$ The main focus of the rehabilitation process should be range of motion strengthening, at both the elbow and glenohumeral joints. ${ }^{8-10,34}$ However, movements or exercise that would place a valgus force on the elbow should be avoided in the beginning. ${ }^{10}$ 
Diagnosing UCL injuries, factors that lead to a torn UCL, and the different surgical techniques are abundant in the literature. However, a comparison of one surgical technique to another or a comparison of return to play rates is scarce. Like other injuries, it is important to compare UCL surgical techniques. Another problem is the limited rehabilitation procedures for the surgeries. Most literature will discuss rehabilitation in one paragraph at the end of a study. This may pose a problem especially for comparison of rehabilitation considerations for a protocol.

With varying surgical techniques one should be able to differentiate and determine which technique would be successful for the athlete. Thus, the following research questions are being asked:

Research Questions

1. What surgical techniques (Jobe, Docking, or newer techniques/variations) are successful in UCL reconstruction?

2. Is there a difference in the return to play rates for different surgical techniques?

3. Is there a surgical technique preference between the different levels of competition (professional or collegiate), or is it strictly physician dependent?

4. What rehabilitation considerations or variations are there for different surgical techniques?

5. Are there different progression, exercises, and throwing progressions in which an athlete returns in a timely manner and between the surgical techniques?

6. What is the return to play rate for UCL injuries treated without surgery?

Experimental Hypotheses

1. The Docking technique will be found to be more successful for surgery and return to play rates. But preference for techniques at each level will be based on the attending physician/surgeon involved.

2. Rehabilitation between different surgical techniques will be slightly similar, with differences found in earlier progressions and more conservative approaches. 
3. Throwing progressions will be found to begin at different time points depending on the surgical technique used.

4. A change in throwing biomechanics will provide for a decreased rate in re-injury.

5. Those who undergo surgery will have a more successful return to play rate and will return to pre-injury levels over those who take on the non-surgical options.

6. There will be enough information in studies to be individually scored on the Coleman Methodology Score.

Assumptions

1. All studies will meet all the inclusion criteria. Inclusion criteria will include:

a. All studies will be written in the English language or translated in the English language.

b. All studies will involve baseball players.

c. All studies will involve collegiate or professional level baseball players.

2. All studies will be able to be scored on a Coleman Methodology Score.

3. There will be enough studies to be scored on the Coleman Methodology Score.

4. Reviewers using the Coleman Method will be reliable.

Delimitations

1. Research found will only apply to baseball athletes.

2. Only studies in English language or an English translation will be used.

3. No studies will meet exclusion criteria.

Operational Definitions

1. Anterior collateral band- Major stabilizer for the medial elbow. ${ }^{8}$

2. Chronic- An injury that typically occurs overtime caused by mircotrauma.

3. Docking technique-Modified surgical technique based off the Jobe technique. Incorporates the use of two tunnels drilled into the ulna and one in the humerus (with 2 exit tunnels). The graft is pulled through the tunnels and docked into place. ${ }^{18,19,33}$

4. Glenohumeral joint- Glenoid fossa of the scapula that meets with the head of the humerus, forming what is called the shoulder joint. ${ }^{35}$ 
5. Jobe technique- Surgical technique made famous by Dr. Frank Jobe, first technique used to reconstruct the UCL on a baseball player. The technique involves 2 tunnels being drilled into the ulna and 2 tunnels into the humerus, which connect. The graft is passed through ulnar tunnels through to humeral tunnels and sutured together. ${ }^{8-10}$

6. Prospective cohort study- A type of study which observations research examines if a specific individual is exposed to a certain factor/disease. The investigator/examiner follows the subject/group of individuals who are exposed to the disease and a control group, which are individuals who are not exposed to the disease, over time. ${ }^{36}$ Level of evidence-II. ${ }^{37}$

7. Randomized control trial- A trial which allows for a difference to be seen between the treatment and control groups. This type of research minimizes bias through a random placement of subjects into control, most through a computer-generated algorithm. Classified as being the highest level of evidence-I. ${ }^{37}$

8. Reconstruction- The use of a tendon graft, autograph or allograft, to form a new ligamentous structure.

9. Reliability- The research being performed is determined to be consistent, through multiple trails and different samples taken from the same population. Also, indicates that instruments used allowed for similar results to be reproduced when used by multiple investigators. 38

10. Retrospective cohort study- A type of study which the investigator will look back in time at studies and records that are archived for a comparison of exposed and non-exposed to determine a difference between the two groups. There is no requirement to follow-up with a subject or follow a subject for an extended period of time. ${ }^{36}$ Level of evidenceIII. $^{37}$

11. Sensitivity- Classification of a subject who has the disease/injury/disorder. High sensitivity indicates a lower rate for a false negative to arise. High sensitivity, with a negative test, allows for the disease/injury/disorder to be ruled out. ${ }^{39}$

12. Ulnar collateral ligament- Located on the medial side of the elbow that provides a valgus support to the elbow. Composed of three band/bundles: anterior, transverse, and posterior. $^{4}$

\section{Limitations}

1. There may be differences between interpretations when scoring the Coleman Methodology Score.

2. There may be studies that cannot be scored on the Coleman Methodology Score.

3. Only studies published in English or an English translation will be included. 
4. Only 3 surgical techniques will be evaluated.

Significance of the Study

Athletes who sustain UCL injuries not only have to consider surgery, but also have an understanding and knowledge of the lengthy rehabilitation process. Like anterior cruciate ligament reconstructions, the process from surgery through return to play is an extensive process that can last anywhere from a few months to a year. Thus, the education of athletic trainers to fully understand this process is paramount.

Research conducted by physicians exists. But the information is limited for athletic trainers in terms of surgery and rehabilitation. Being able to provide a bridge between physicians and athletic trainers should be considered. Conducting this study to provide a link for athletic trainers will not only help with their knowledge of UCL injuries, but also aid athletic trainers in the ability to prevent this injury.

Aside from an education standpoint, providing a study for athletic trainers on the surgical techniques used for UCL injuries and what each surgery is, the time an athlete might miss and the rehabilitation process for each surgery is important as well. When surgical techniques change, the rehabilitation will also change. As physicians will have their own guidelines, a personal preference may be evident. These variations in surgeries will lead to variations in rehabilitation as well as the length of time an athlete will be out. In addition, throwing progression time frames and the succession of athletes returning to play are varied. Another concern is conservative methods used where rehabilitation occurs before surgery options are explored.

To benefit athletic trainers, a bridge between the research and physicians is important. UCL injuries are commonplace in the baseball world. Thus, providing a study on injuries athletic trainers may not have much control over will benefit by providing ways to tailor rehabilitation 
for different surgical techniques. Being able to provide research to educate athletic trainers is paramount. Education is crucial starting with athletic trainers. They in turn can educate the coaching staff, strength and conditioning staff, and athletes on the importance of prevention and concerns when the injury occurs.

It is important that this information is disseminated to athletic trainers and other clinicians. This would be a topic for inclusion in journals and other publications. Being able to present the information found in the study in the form of workshops and presentations at various state, district meetings/conventions, and at the national level is another alternative. The main focus of the study should be to bridge the gap between the physicians research and relate this to the athletic training profession. 


\section{APPENDIX B}

\section{LITERATURE REVIEW}

\section{INTRODUCTION}

Baseball injuries are quite common. When it comes to baseball and injuries, injuries to the glenohumeral joint or sprains/strains are evident. In more recent years, ulnar collateral ligament (UCL) injuries have increased. UCL injuries in the past resulted in as being a careerending injury. Individuals on the team who may sustain a UCL predominantly are pitchers. Twenty-six and three tenths percent of pitchers will sustain an elbow injury over infielders and outfielders. $^{2}$ Over the years, knowledge of these injuries has increased as well as treatment options.

Over 40 years ago, Dr. Jobe was the first physician to reconstruct a UCL injury. This provided a viable option for those who sustained a UCL injury. ${ }^{8}$ From advances in surgery to a better understanding of throwing/pitching biomechanics, UCL injuries are evolving into an injury which the damaged ligament can be reconstructed, but entails an extensive rehabilitation process. A typical UCL injury will place an athlete on the disabled list anywhere from 12-18 months. $3,4,8,10,13,18,20,21$ This will vary based on the level of competition, the mechanism of injury and the physician treating the injury.

The injury usually occurs during the late cocking phase/early acceleration phase of

throwing. ${ }^{3-5,9-14}$ There are numerous modifications and variations for the Jobe technique, but only two are evaluated in this study. The Jobe technique and the Docking technique are the two more common techniques used for UCL reconstruction. These techniques are both similar and different. The primary similarities are the autografts used and rehabilitation followed postoperatively. ${ }^{3,6,8,10,13,21,22}$ The primary differences are the number of tunnels drilled (two 
connecting tunnels ${ }^{8,10,13}$ versus one connecting tunnel ${ }^{3,21,22}$ ), the graft end results (“figure- 8 ", ${ }^{\prime, 10,13}$ versus "Y-shaped" $" 19$ ), and transposing of the ulnar nerve. . $^{8,10,13}$

Success rates for these two surgical methods vary with a $68 \%$ return to play rate for the Jobe technique ${ }^{10}$ and a $92 \%$ for the Docking technique. ${ }^{22}$ The Docking technique has better outcomes but also a decrease in complications post-operatively. ${ }^{22}$ The major headers included in this literature review are elbow anatomy, epidemiology, etiology, non-surgical treatment, surgical treatment, rehabilitation and return to play.

Elbow Anatomy

When trying to understand how an injury occurs to determine treatment, understanding the anatomy is important. The elbow is not

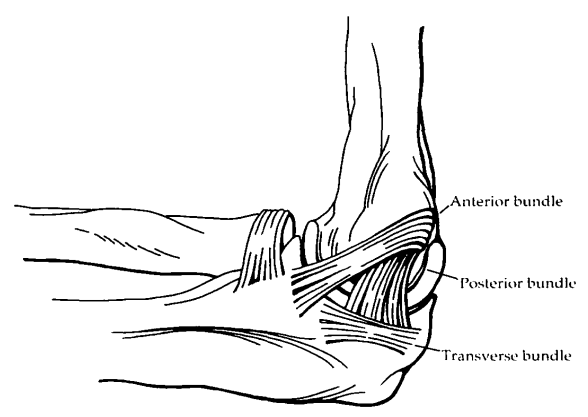

considered to be a very complex structure. Three

bones form the joint and consist of the humerus, ulna

Figure B1. Medial elbow UCL bundle ${ }^{13}$ and radius. ${ }^{7,40}$ Two major muscle masses that originate off the epicondyles are the flexor and extensor muscle mass. ${ }^{7,40}$ The flexor mass consists of: flexor carpi ulnaris, plamaris longus, flexor digitorium superficialis, pronator teres, and flexor carpi radials. ${ }^{3,7}$ Ligaments stabilize the elbow on the lateral and medial side. The UCL is located on the medial aspect of the elbow. ${ }^{5}$ Due to general functional anatomy, the elbow is considered to be the weakest link in the kinetic chain. ${ }^{12}$

The UCL is not a singular ligament; it is composed of three different ligaments. These ligaments are the anterior bundle, posterior band and the transverse ligament. ${ }^{3-7,40}$ Of these three ligaments, the anterior and posterior ligaments are the only ones to be considered functional with motion. ${ }^{5}$ 
The anterior bundle ligament is composed of the anterior and posterior bands. ${ }^{3-7,40}$ The bundle's location, in relation to the medial aspect of the elbow, is where the bundle originates from the inferior medial epicondyle and the insertion point on the coronoid process, on the sublime tubercle. ${ }^{3,4,5,6,40}$ This bundle is considered to be the primary stabilizer of the medial elbow, ${ }^{3,4,6,9,11,13,40}$ and stabilizes the elbow through a flexion/extension arc between $30^{\circ}-120^{\circ} .{ }^{4,6,7}$ This bundle of ligaments provides a valgus support for the elbow. ${ }^{3-5,7,40}$ During elbow extension, the anterior band is taut. ${ }^{3,7}$ The posterior band is considered to be more isometric with movement, and stabilizes from $60^{\circ}$ of extension through full elbow flexion. ${ }^{3}$ During flexion, the posterior band is taut. ${ }^{3}$ When an injury occurs to the elbow and is diagnosed as a torn UCL, the anterior band of the anterior bundle is most commonly torn.

The posterior ligament is anatomically thinner than the anterior, which makes it weaker than the anterior bundle. ${ }^{4}$ The ligament is more of a fan shaped ligament. ${ }^{6}$ The origin is the medial epicondyle of the humerus and insertion is on the olecranon of the ulna. ${ }^{5,6,40}$ During movement, especially flexion, the posterior ligament stabilizes the elbow from $90^{\circ}$ flexion through full flexion. ${ }^{4}$ It has been reported that the posterior ligament contributes little to no valgus stability to the medial elbow. ${ }^{40}$ This could be one reason why this ligament is not commonly torn, over the anterior band of the anterior bundle, during a UCL injury. However, this ligament has a primary role in providing stability for the posteromedial rotatory instability of the elbow. ${ }^{40}$

The final ligament of the UCL is the transverse ligament. The transverse ligament is commonly referred to as the oblique or cooper's ligament. ${ }^{4,6,7}$ The ligament originates from the medial coronoid process and inserts on the medial aspect of the olecranon. ${ }^{6,40}$ This ligament is unique because it does not technically cross a joint line. ${ }^{4}$ 


\section{Epidemiology}

The prevalence of injuries to the upper extremity in baseball account for about $45 \%$ of

the injuries. ${ }^{1}$ With professional baseball players, upper extremity injuries will affect $>50 \%$ of the athletes at some point in their career. ${ }^{12}$ Of these injuries to the upper extremity, the glenohumeral joint has a higher rate of injury, followed by the elbow. A common injury to the elbow is a torn UCL. This injury does not occur nearly as often as an injury to the glenohumeral joint, but can be just as debilitating to an athlete. ${ }^{2}$

Mostly pitchers sustain elbow injuries to the UCL. Pitchers have a higher injury rate (34\%) over fielders. ${ }^{1,4,6,13}$ Twenty-six and three tenths percent of pitchers sustained elbow injuries during one season. ${ }^{2}$ Most of the causes may be related to repetitive throwing, various velocities, or poor mechanics. ${ }^{12,15}$ However, injury can occur over multiple seasons or acutely. ${ }^{5,6,9,12,14,15}$ The mechanism, of these injuries could be related to risky behaviors, throwing through the pain or by trying to increase velocity. ${ }^{1}$ This could lead to further injury to the elbow and/or compensation in other locations.

The most common time frame for a UCL injury is during the first few months of the season, including spring training. ${ }^{1,2,9}$ As this injury requires an athlete to be on the disabled list for a minimum of 3-4 months, a pitcher who sustains an elbow injury could potentially miss most of the season, if not the entire season. ${ }^{2}$

\section{Etiology}

UCL injuries occur due to multiple intrinsic and extrinsic factors. A UCL can rupture from mircotears caused over time from repetitive throwing. ${ }^{8-10,12,14,15}$ This would classify the injury as being more chronic, or overuse injury in nature. ${ }^{5,6,8-10,12,14}$ However, there is still a chance that an injury to the UCL can be considered acute. This occurs when a pitcher begins 
throwing and describes a "pop" followed by pain.9,10,13,14 The athlete will begin to feel and complain of the elbow feeling unstable and likely not continue to throw. ${ }^{14}$

Aside from a UCL tearing due to overuse and micro tears, the UCL will tear due to abnormalities during the biomechanics of throwing. ${ }^{15}$ When an athlete is throwing a ball, the athlete will move through 5 phases of throwing. These phases are wind-up, cocking (which is split into early cocking and late cocking), acceleration, deceleration and finally the follow through phase. ${ }^{4}$ It has been found that an injury to the UCL does not occur randomly through the throwing phase. When a UCL injury occurs, it is mostly during the late cocking and acceleration phase..$^{3-5,9-14}$ One study reported on pitchers who sustained a UCL injury during the follow through phase of throwing, but this is considered rare. ${ }^{9}$

The cocking phase is defined as lead foot contact (from the wind up) to maximum shoulder external rotation. ${ }^{4,12}$ In more detail, the motion that occurs at the glenohumeral joint during the cocking phase is movement mostly from the glenohumeral joint and elbow. The elbow will flex from $90^{\circ}-120^{\circ}$, while at the same time the forearm will pronate $90^{\circ}{ }^{4}$ At the glenohumeral joint, the joint will abduct and externally rotate from a range of $90^{\circ}$ up to $180^{\circ}$. Once both of these movements occur at the glenohumeral and elbow joints, the cocking phase is considered over, and the acceleration phase will begin.

The acceleration phase follows the cocking phase with maximum glenohumeral external rotation to ball release. ${ }^{4,12}$ Once maximum external rotation at the glenohumeral joint and maximum flexion at the elbow occur the acceleration phase begins. The elbow joint begins to extend, while the glenohumeral joint begins to adduct and internally rotate. ${ }^{4}$ The phase ends once the ball is released from the throwing hand. ${ }^{4}$

As the elbow is flexed through an arc from $90^{\circ}-120^{\circ}$ and begins to forcefully extend, there is a moment of valgus stress placed upon the elbow. ${ }^{4,5,10,12}$ It is this extreme valgus moment 
where $64 \mathrm{Nm}$ is placed on the UCL. ${ }^{4-6,11}$ This force is doubled the normal amount $(34 \mathrm{Nm})$ of force the elbow can handle without causing injury. Anz et al. ${ }^{12}$ reported that a baseball player's elbow could sustain a valgus stress up to $74.70 \mathrm{Nm}$. After that, the UCL failed when the forces exceeded $91.62 \mathrm{Nm}$. This is drastically different compared to what researchers have found with the valgus moment of $64 \mathrm{Nm}$ causing UCL tears. This could be related to the anatomic differences and the ability to handle various forms of stresses differently. One mechanism of the injury that is evident in all that sustain an injury to the UCL is the greater valgus stress placed on the elbow along with greater glenohumeral external rotation. ${ }^{12,115,16}$ Elbow valgus stress/forces peak when the glenohumeral joint is in maximal external rotation. ${ }^{12}$

Structures that surround the elbow joint can become fatigued, and leading to UCL injuries. When a pitcher is throwing in a game, there is a tendency to throw faster and harder when warranted. This leads to fatigue of the flexor masses that originate on the medial side of the elbow. ${ }^{5,6}$ When this fatigue occurs, abnormal biomechanics become evident and a decrease in flexibility is noted. ${ }^{5}$ The muscles become over worked or overloaded, and an imbalance occurs. This change in throwing mechanics places more stress on the UCL. ${ }^{5,6}$

In baseball, the number of pitches thrown and the types of pitches thrown are recorded. This can be controlled as there is a link between pitch count, velocity and type of pitch with UCL injuries. $^{6,13}$ The harder and faster a pitcher throws and the longer he is kept in the game, the chances for an injury to the UCL increase. Pitchers are three-times more likely to sustain an injury during a game than in practice. ${ }^{1}$ When a pitcher becomes fatigued throwing mechanics become compromised, which will increase the stress placed upon the elbow. 


\section{Non-Surgical Treatment}

Treatment of UCL injuries may begin following a non-operative approach. Usually physicians' would recommend 3 months of rehabilitation and pain management treatments before a surgical option is explored. , $^{3,4,8-10}$

During this time of active rest and rehabilitation, modalities are used for pain management. It is suggested that cryotherapy be used and NSAIDs as the first line of defense, with rest from activity. ${ }^{3-6,10}$ Aside from the use of modalities to conservatively treat UCL injuries, exercises are often implemented. Range of motion (ROM) and strengthening exercises for all the structures that surround the elbow and glenohumeral joint are initiated. ${ }^{3-6,8,10}$, The exercises should focus on restoring the ROM at the elbow and strength back to normal and painfree motions. ${ }^{3,4,10}$ These exercises should also avoid valgus stresses in the beginning of rehabilitation. ${ }^{10}$ The exercises should be isometric in the beginning of the rehabilitation program, and eventually progress to isotonic, once ROM and exercises are pain-free. ${ }^{4}$ Depending on the pain and what causes pain, some physicians' may place the athlete in a hinge brace, to prevent full extension of the elbow. ${ }^{4}$

Once ROM and strength in the affected arm are restored back to normal and pain-free, a throwing progression may begin. ${ }^{3,10}$ In the beginning of the throwing progression, a biomechanical evaluation should be completed. ${ }^{5,10}$ It is suggested that if biomechanics are corrected early enough, the chances for re-injury and/or surgery being necessary decresases. ${ }^{10,12,15}$

Once near the end of the 3-month period of conservative treatment, if the athlete is pain free and able to adequately throw, then the athlete may begin a return to play progression and return to competition. ${ }^{4}$ Meyer et al. ${ }^{3}$ reported a success rate of $42 \%$ of the athletes being able to return to play, within 3 months, without surgery. 
The contraindication to this conservative treatment is the athlete may not improve. This means that the pain cannot be controlled, throwing cannot be completed successfully without pain or fatigue occurring, and ROM is not fully restored. When this happens, the only option for the athlete is to have surgery. ${ }^{10}$

Surgical Treatment

Physicians' will sometimes choose the surgical route first over 3 months of rehabilitation and biomechanical changes. ${ }^{6,10,14,15,20}$ The exception to avoiding the conservative route is when the UCL acutely tears. ${ }^{3}$ When this occurs, it is recommended that surgery should take place within 2 weeks of initial injury. ${ }^{3}$ Some physicians even consider surgery to be the gold standard for treatment of UCL injuries. ${ }^{8,20}$

Other indications for surgery, aside from an acute tear and failure of a conservative route, is when are the athlete wants to return to sport and possibly progress up in competition. ${ }^{13}$ Usually if laxity is found, which causes pain and instability, surgery is considered. ${ }^{14}$ The two surgical techniques that are commonly used to reconstruct the UCL for a baseball athlete are the Jobe and Docking technique.

Jobe technique: Dr. Frank Jobe was the first surgeon to develop a reconstruction for the UCL. One of three possible grafts are used from the ispilateral or contralateral limb of the athlete. ${ }^{8,9}$ The palmaris longus, plantaris and/or toe extensor tendons are used (refer to Table B1). ${ }^{8,10,13}$

The athlete is prepped for surgery and put under general anesthesia. The athlete is supine and the arm is placed abducted on an arm board or in a tourniquet. ${ }^{8-10,13}$ Working from the medial aspect of the elbow, an incision is made from the medial epicondyle to the medial aspect of the forearm. ${ }^{8-10,13}$ The incision makes a semilunar curve and is only about $10 \mathrm{~cm}$ in length. ${ }^{8,9}$ Once the flexor mass is exposed, an incision to longitudinally spilt the muscle is used to expose the 


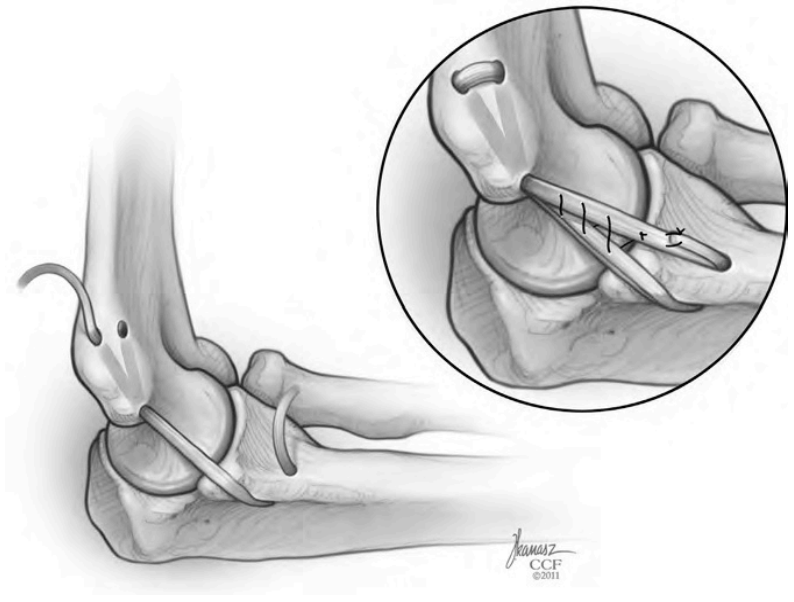

Figure B2. Jobe technique graft placement and attachment ${ }^{4}$
UCL. ${ }^{8}$ In the process, the pronator teres is transected and reflected to allow for more room to work and expose the full UCL. ${ }^{8}$ Once these muscles are exposed and moved accordingly, the ulnar nerve is transposed if found to be in the way of the UCL (Refer to Table B1 for studies that transposed the ulnar nerve) ${ }^{8,9,13}$

Once the UCL is fully exposed and the old ligament is cleared out, the surgeon drills two holes into the medial epicondyle and ulna, just off the distal tubercle. ${ }^{8,9,13}$ These holes are drilled, to create one tunnel in each bone. ${ }^{13}$ As these tunnels are created, the graft is prepped to pass through the tunnels. The graft is passed from the tunnel hole in the ulna, through the humerus, through the full tunnel and is pulled taut and connected with sutures in the middle. ${ }^{8,9,13}$ This pattern creates a "figure- 8 " in the graft. ${ }^{8,9,13}$ The new UCL, once in place and secure, the ulnar nerve (if transposed), pronator teres and flexor mass are all placed in correct anatomical position and secured. The surgical incision is sutured closed. ${ }^{8,9,13}$ Before the surgery was considered to be a success, the surgeon will take the elbow through a few degrees of ROM and also tests varus stability. ${ }^{8,9,13}$ Once the surgeon is pleased with the new UCL, the athlete is placed in a splint flexed to $90^{\circ}$ to immobilize the elbow. $3,4,8,9,13$

Athletes who underwent the Jobe technique had a success rate of less than $85 \% .^{8-10,13}$ When the results were first reported in 1986 , only a $62 \%$ success rate was reported for athletes returning to baseball. ${ }^{8}$ In 1992 , it was reported that the success rate increased to $68 \%$ of athletes returning. ${ }^{9}$ More recent studies using the Jobe technique reported success rates that were 
higher. ${ }^{8,9,13}$ One study even reported a success rate of $83 \% .{ }^{10}$ Table B1 reports more outcome rates for Jobe technique studies.

With all the new surgical techniques, there are always some concerns. Dodson et al. ${ }^{21}$ expressed concern with the use of the Jobe technique for the strength of the graft. The concern expressed was in the Jobe technique, the graft was just sutured together, whereas in the Docking technique the graft is docked into the tunnels. ${ }^{21}$ There was also some concern over the detachment of the flexor mass during the surgery, the large tunnels created, and nerve complications due to transposing the ulnar nerve. All of these concerns lead to the creation of the Docking technique.

Docking technique: Anytime a new surgical technique is developed, surgeons alter the methods for better outcomes and to decrease the chance for complications. ${ }^{21}$ The leading surgeon to create the Docking technique is Dr. Rohrbough. ${ }^{22}$ This technique uses one of three tendon grafts from the ipsilateral or contralateral limb. These grafts are the gracilis, palarmis longus, and toe extensor, with the palmaris longus used most often (refer to Table B2). ${ }^{3,6,21,22}$ The athlete is prepped the same way as the Jobe technique, with the use of anesthesia. The athlete is positioned supine, with the arm in a tourniquet or abducted on an arm board, with mild varus stress at the elbow. ${ }^{3,6,21,22}$

Once the athlete is placed in an optimal

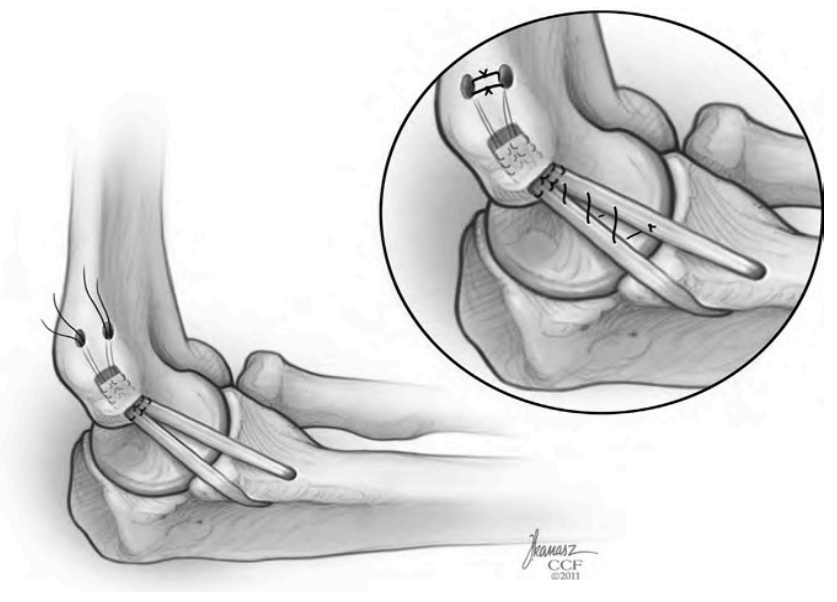

Figure B3. Docking technique graft placement and attachment ${ }^{4}$

position, a $8-10 \mathrm{~cm}$ incision is made from the medial epicondyle to about $2 \mathrm{~cm}$ beyond the sublime tubercle. ${ }^{3,6,21,22}$ Once the flexor muscles are exposed, some surgeons may use a muscle splitting technique from here. (Refer to Table B2 for studies that split the muscles) This would 
split the posterior third of the pronator teres and the anterior portion of the flexor carpi ulnaris. ${ }^{3,21,22}$ When the muscles are split, exposing the UCL, tunnels are drilled in the anterior and posterior sublime tubercle of the ulna and on the anterior side of medial epicondyle of the humerus. ${ }^{3,21,22}$ An exit tunnel is created from the tunnel in the medial epicondyle to the intramuscular septum. ${ }^{3,21,22}$ The graft is then passed through the tunnels in the ulna and up to the humeral tunnel. Once the graft is at the exact location measured out to be the length needed through the exit tunnel, the graft is docked in place. ${ }^{21,22}$ An Endobutton is used to secure the graft in the exit tunnel. ${ }^{3}$ The graft is then secured down and the elbow is sutured closed once all the structures are in the anatomical position. ${ }^{3,21,22}$ Before the surgeon is finished, the elbow is moved through ROM and a varus stress is applied to test the ligament. ${ }^{3,21,22}$ Upon the completion of the surgery, the athlete is placed in a splint to immobilize the elbow at $90^{\circ} .{ }^{3,4}$ Rohrbough et al. ${ }^{22}$ suggested that the athlete be placed in a plaster splint with the elbow flexed to $60^{\circ}$.

One major difference between this surgical technique and the Jobe technique is that the ulnar nerve is not transposed. The form the graft takes on is also different. In the Jobe technique, the graft forms a "figure-8" whereas in the Docking technique, the graft creates more of a "Yshape". 19

Comparing the Docking technique to the Jobe technique, there are higher success rates for an athlete to return to previous levels of competition. Multiple studies have reported a success rate of $90 \%$ or better for the Docking technique. ${ }^{18-22}$ These results lead to allowing athletes to return back to throwing, but there was no difference noted in the timeline for return to play. Refer to Table B2 for outcome rates for specific studies. 
Table B1. Jobe Surgical Technique

\begin{tabular}{|c|c|c|c|}
\hline Authors & Surgical Procedure & Outcome & Conclusion \\
\hline $\begin{array}{l}\text { Azar et al } \\
2000\end{array}$ & $\begin{array}{l}\text { - Modifications made: ulnar nerve transposed and } \\
\text { flexor mass not detached } \\
\text { - Graft used- Palmaris longus ( } 63 \text { patients), } \\
\text { extensor tendon ( } 9 \text { patients), plantaris ( } 6 \text { patients) } \\
\text { - } 2 \text { tunnels drilled in medial epicondyle and } 2 \\
\text { drilled in ulna } \\
\text { - Graft passed in "figure- } 8 \text { " from ulnar to humerus, } \\
\text { sutured together }\end{array}$ & $\begin{array}{l}\text { - } 53 \text { of } 67 \text { patients returned to previous level } \\
\text { of competition ( } 79 \% \text { RTP rate) } \\
\text { - } 73 \% \text { of professional baseball players } \\
\text { returned } \\
\text { - } 10 \text { were unable to RTP at same level (went } \\
\text { down a level) } \\
\text { - } 4 \text { did not return at all } \\
\text { - } \text { RTP average- } 9.8 \text { months } \\
\text { - Professional baseball players RTP- 1 year }\end{array}$ & $\begin{array}{l}\text { - Good results were concluded } \\
\text { from this study with good RTP } \\
\text { rates at any level of competition. } \\
\text { - Transposition of the ulnar nerve } \\
\text { was found to have fewer } \\
\text { complications }\end{array}$ \\
\hline $\begin{array}{l}\text { Bennett et al. }{ }^{14} \\
2009\end{array}$ & $\begin{array}{l}\text { - Hybrid technique used- based off Jobe technique } \\
\text { - Modifications: places graft in a more anatomical } \\
\text { position and uses screw fixations } \\
\text { - Graft used- Palmaris longus or semitendinosus } \\
\text { - Flexor mass incised } \\
\text { - Three humeral tunnels drilled } \\
\text { - Grafts passed through the tunnels, creating a loop } \\
\text { through two tunnels } \\
\text { - Ulnar tunnel drilled and one end of graft is pulled } \\
\text { through this tunnel. } \\
\text { - Optimal tension is obtained and graft is sutured } \\
\text { down using screw fixations }\end{array}$ & - No outcomes reported & - No conclusion reported \\
\hline $\begin{array}{l}\text { Cain et al. } \\
2010\end{array}$ & $\begin{array}{l}\text { - Modified Jobe technique } \\
\text { - Arthroscopy used to confirm diagnosis } \\
\text { - Graft used- Palmaris longus ( } 935 \text { patients), toe } \\
\text { extensor tendon ( } 30 \text { Patients), gracilis ( } 294 \\
\text { patients) } \\
\text { - Ulnar nerve transposed }\end{array}$ & $\begin{array}{l}\text { - } 743 \text { of } 942 \text { patients followed-up }(79 \%) \\
\text { - } 83 \% \text { RTP to previous level of } \\
\text { competition/higher } \\
\text { - } 75.5 \% \text { RTP to Major League Baseball } \\
\text { - } 67 \% \text { RTP to collegiate baseball } \\
\text { - } 83 \% \text { RTP for high school baseball } \\
\text { - } 18 \% \text { did not return } \\
\text { - Return to throwing- } 4.4 \text { months } \\
\text { - RTP average- } 11.6 \text { months }\end{array}$ & $\begin{array}{l}\text { - } 83 \% \text { of athletes returned to their } \\
\text { previous level of competition with } \\
\text { UCL reconstruction using the Jobe } \\
\text { technique } \\
\text { - There was a higher RTP rate for } \\
\text { the Major League Baseball players } \\
\text { over other levels. }\end{array}$ \\
\hline $\begin{array}{l}\text { Conway et al. } \\
1992\end{array}$ & $\begin{array}{l}\text { - Graft used- Palmaris longus ( } 35 \text { patients), } \\
\text { plantaris ( } 7 \text { patients), toe extensor tendon (1 } \\
\text { patient) }\end{array}$ & $\begin{array}{l}\text { - } 68 \% \text { RTP at previous level or higher of } \\
\text { competition w/ UCL reconstruction } \\
\text { - } 13 \% \text { RTP at a lower level of competition w/ }\end{array}$ & $\begin{array}{l}\text { - Chance for ulnar nerve } \\
\text { complications with the surgery, } \\
\text { when ulnar nerve transposed, was }\end{array}$ \\
\hline
\end{tabular}


- Ulnar nerve transposed

- Flexor mass split and moved from medial

epicondyle

- 2 tunnels drilled in ulna and humerus

- Graft passed in "figure-8" and sutured together.

Jobe et al. ${ }^{8}$ 1986
- Graft used- Palmaris longus (12 patients), plantaris (3 patients), achilles tendon (1 patient)

- Flexor mass split

- Ulnar nerve mobilized

- Four tunnels were drilled; 2 in the ulna, 2 in the humerus

- Graft passed through tunnels in "figure-8" and sutured together
UCL reconstruction

- $5 \%$ did not return

- 10/16 patients RTP at previous level or higher of competition (62\%)

- 1 went down a level

- 5 retired from the MLB, but this was not due to the surgery

- RTP- 12-18 months only $21 \%$, leaving only $3 \%$ not returning due to these complications

- RTP- 1 year post-op

- Pitchers RTP-18-24 months before pitching half a game

- First surgery done on reconstructing the UCL in the elbow.

- Patients were able to return to pitching between 11 and 19 months

- Strength was noted to improve 2 years post-op

- With lengthy rehabilitation for this type of surgery, patients who are motived and not easily

discouraged seemed to return more successfully.

\section{Table B2. Docking Surgical Technique}

\begin{tabular}{|c|c|c|c|}
\hline Authors & Surgical Procedure & Outcome & Conclusion \\
\hline $\begin{array}{l}\text { Bowers et al. }{ }^{20} \\
2010\end{array}$ & $\begin{array}{l}\text { - Graft used- Palmaris longus or gracilis } \\
\text { - Medial incision from proximal medial epicondyle to } 2 \\
\text { cm past sublime tubercle } \\
\text { - Flexor mass split } \\
\text { - Ulnar nerve transposed (when it can not be protected) } \\
\text { - Connecting tunnels drilled in sublime tubercle of ulna } \\
\text { - Two tunnel drilled in medial epicondyle } \\
\text { - Exit tunnel was drilled through anterior-aspect of the } \\
\text { epicondyle } \\
\text { - One end of the graft is pulled through ulnar tunnel } \\
\text { while the other end is pulled medial epicondyle tunnels. } \\
\text { - One end of the graft pulled through the humeral exit }\end{array}$ & $\begin{array}{l}\text { - } 21 \text { baseball players received the } \\
\text { surgery } \\
\text { - } 19 \text { players RTP within } 1 \text { year at } \\
\text { previous competition level }(90 \%) \\
\text { - } 2 \text { players RTP at lower levels of } \\
\text { competition (were still able to throw } \\
\text { though) }\end{array}$ & $\begin{array}{l}\text { - With } 90 \% \text { RTP in this study, it can } \\
\text { be reported that the docking } \\
\text { technique is a successful technique } \\
\text { to use. } \\
\text { - It allows for an athlete to RTP } \\
\text { within one year, and with successful } \\
\text { rehabilitation, the athletes returned } \\
\text { to their previous level of } \\
\text { competition. } \\
\text { - All athletes were cleared to throw } \\
\text { and compete, even though } 2 \text { did not } \\
\text { return to competitive playing. }\end{array}$ \\
\hline
\end{tabular}


Dodson et al. ${ }^{21}$

2006

Meyer et al. ${ }^{3}$ 2008
- Graft used- Palmaris longus or gracilis

- Flexor mass split

- Two connecting tunnels drilled in ulna

- One tunnel drilled in the humerus

- Graft passed through ulnar tunnels, and docked into humeral tunnel

- Graft docked in place and secured down

- Graft used- Palmaris longus, toe extensor and plantaris

- Arthroscopy used to diagnosis and rid loose bodies

- Flexor mass split

- Graft docked into two tunnels drilled into sublime tubercle

- Two connecting tunnels drilled in medial epicondyle of humerus, with 2 exit tunnels drilled

- Graft passed up though humerus tunnels and then through exit tunnel.

- Endobutton used at end of graft pulled through exit tunnel

- Graft docked in place and secured down

Rohrbough et al. ${ }^{22}$ 2002
- Graft used-Palmaris longus (34 patients), gracilis (1 patient), toe extensor (1 patient)

- Ulnar nerve transposed-only if history of nerve problems are present before surgery

- Diagnostic arthroscopy used

- Flexor mass split

- Two connecting tunnels drilled in sublime tubercle of the ulna

- One tunnel is drilled in the medial epicondyle, with 2 small exit tunnels drilled

- Graft is passed from the ulnar tunnels up through to humeral tunnel and out through an exit tunnel

- Graft docked into tunnels and sutured down via exit tunnels.
- 100 patients followed up

- $90 \%$-RTP at previous level of competition or higher

- $7 \%$ moved down a level of competition

- $1 \%$ went down to recreational level

- $2 \%$ did not return

- No outcomes reported

- 21/36 returned to previous level of competition

- $12 / 36$ advanced to competing at higher levels

- $92 \%$ RTP rate
- This technique used validates that majority of the patients who undergo UCL reconstruction will return to play at previous level of competition. $97 \%$ RTP rate

- Graft fixation shows largest gap, which is chosen by the surgeon

- No clinical trails have been performed to compare
- None of the patients who under went the docking technique did not fail to RTP at previous level or higher at the collegiate and professional level

- Only downfall to technique noted was the number of tunnels drilled in the humerus (4 total)

- High RTP rate for athletes who receive UCL reconstruction using the docking technique 


\section{Rehabilitation}

The rehabilitation process for UCL injuries post-operatively is deemed to be fairly extensive. When an athlete has surgery for UCL reconstruction, the timeline of rehabilitation can last anywhere from 12 to 18 months. Most rehabilitation protocols are divided into 4 phases, depending on the surgery and technique used. There is some overlap when comparing the phases physician to physician. It can also be noted that one progression may come sooner or later compared to others. (Refer to Table B3 and Table B4 for phase breakdown by weeks/months or studies that suggested no timelines.)

Phase one typically lasts from day 1 post-op to about 3 weeks post-surgical. ${ }^{3-5,8-10,13,21,22}$ Week 1 consisted of the athlete remaining in a hinge brace, flexed to $90^{\circ}$ for 10 days. $3,6,9,13,14,18$ The athlete is able to begin, and encouraged to begin, wrist and hand ROM exercises and to work on gripping or squeezing a ball..$^{3,4,6,8,9,13,22}$ For ROM allowed during phase one, there is some controversy over what the surgeons are comfortable with and how much ROM is allowed. Some surgeons report that $\mathrm{ROM}$ is allowed from $30^{\circ}$ extension to $100^{\circ}$ flexion. ${ }^{3,13}$ Other surgeons are more conservative with only allowing $30^{\circ}$ extension to $90^{\circ}$ flexion. ${ }^{14,18,20,21}$ There are also some surgeons who will only allow for $40^{\circ}$ extension to $100^{\circ}$ flexion, ${ }^{4}$ or $45^{\circ}$ extension to $90^{\circ}$ flexion. $^{22}$ (Refer to Table B3 and Table B4 for study prefers on ROM allowed.) However, there are no difference noted when comparing the ROM's allowed between the Jobe and Docking technique. Beginning at the end of week 2 and going into week 3, the athlete is permitted to begin isometric strengthening exercise for the glenohumeral joint and arm, and continue with ROM exercises of the glenohumeral joint and elbow. ${ }^{3,9,13,14,18}$ The brace is also opened up to allow for motion from $15^{\circ}$ of extension to $105^{\circ}-110^{\circ}$ of flexion. ${ }^{3,13,14,21}$ From this point on, the $\mathrm{ROM}$ is increased in the brace from $5^{\circ}$ of extension and $10^{\circ}$ of flexion each week until full ROM is achieved. ${ }^{3,13}$ 
Cain et al. ${ }^{10}$ suggested that in order to progress to phase two, full ROM needs to be restored within the first three weeks of rehabilitation. There is also a suggestion that rehabilitation should not begin until 6 weeks post-op. ${ }^{21,22}$ (Refer to Table B4 for studies with suggestions on waiting to begin rehabilitation.)

Phase two begins at week 4 and goes through week $8-10 .^{3,4,6,8-10,13,21,22}$ During this phase, full ROM should be restored by week 6 and exercises can begin to progress from isometric to isotonic. $3,4,10,13,14,20$ The focus of the exercises during this phase is strengthening of the glenohumeral, elbow, wrist and rotator cuff. ${ }^{6,8,9,13,14,20}$ By week 8 , light weights may begin to be incorporated into the strengthening exercises. ${ }^{4,8,13}$ The weights are allowed to progress by 1 pound per week, until the end of week $8 .{ }^{\text {Azar }}$ The brace is discontinued by week 8 as well..$^{3,13}$ During this phase, it is suggested that a Thrower's 10 Exercise Program begin. ${ }^{10}$ It is important that during this phase, any exercises that would place a valgus stress on the elbow are avoided until 4 months post-op. ${ }^{4,6,9,22}$

Phase three is considered to be the advanced phase in the rehabilitation starting with weeks $9 / 10$ and ending at 12-36 weeks. , $^{3,6,8-10,13,21,22}$ It is during this phase when sport-specific exercises may begin..$^{3,4,9,10,13}$ Stretching and flexibility programs are encouraged during this phase. ${ }^{13}$ The focus of the strengthening exercises are the same as phase two but a little more aggressive for glenohumeral, rotator cuff, elbow, and forearm/wrist. ${ }^{4,13,21,22}$ It is during this phase when internal and external rotation at the glenohumeral joint should be restored. ${ }^{4,10}$ Dynamic stabilizations for the glenohumeral joint and elbow are encouraged to begin during this phase as well. ${ }^{13}$ Week 12 is when plyometric exercises and an upper extremity lifting program are allowed to begin. ${ }^{4,10,13,18,21}$ During this phase, Bennett et al. ${ }^{14}$ suggested that during week 12 it would be appropriate for light weight strength exercises to begin. During this phase, full body (core and lower extremity) should be allowed to take place in a strengthening program. ${ }^{10,18}$ 
Phase four is usually considered the last phase. This phase typically goes from weeks 1352 and on through return to play progression. ${ }^{3,10,13}$ Some physicians may divide this phase into a fifth phase, which would be when throwing progressions are allowed to begin.

There is some controversy on when to begin the interval-throwing progression. Some feel that light tosses up to 45 feet in distance are acceptable around week 14 post-op, ${ }^{13,14}$ whereas others feel no throwing should be allowed during the first 4 months. ${ }^{3,4,9,10,14,18,20-22}$ Eventually the tosses will progress to throws with 180 feet allowed for distance. ${ }^{13}$ The throwing days are suggested to occur every other day. ${ }^{21}$ The only time an athlete is able to increase the throwing distance is when in the previous phase throwing is painfree. ${ }^{3,13}$ Conway et al. ${ }^{9}$ suggested that a throwing progression begin 4 months post-op. Specifics for progression with throws are tossing the ball 30-40 feet, 2-3 times per week for about 10-15 minutes each session. ${ }^{9}$ If pain occurs at all during this interval-throwing program, the athlete is to stop for the day, and follow the previous day protocol. ${ }^{21}$ Jobe et al. ${ }^{8}$ has a different philosophy on pain when throwing. It is noted that when pain begins, to stop throwing for two weeks and then slowly progress back into the throwing phase. ${ }^{8}$ If the athlete begins to throw earlier than 4 months, it is suggested that a throwing biomechanical analysis should occur around week $10{ }^{4}$

Month 5 allows for the ball to be tossed up to 60 feet with strengthening exercises in rehabilitation still continuing. ${ }^{6,9}$ Month 6 allows for lob throws from 60 feet with an easy windup. ${ }^{6,9}$ It is during this month when throwing days and rehabilitation exercises alternate days. ${ }^{9}$ Throwing continues to be on flat ground during this month. ${ }^{22}$

Around month 7 or 8 , pitchers will be able to begin the progression of throwing up to 120 feet off the mound, then on the mound. ${ }^{9,13,14,20,22}$ The pitcher is allowed to throw up to speeds of $75 \%$ of maximum velocity. ${ }^{9}$ Dodson et al. ${ }^{21}$ suggested that pitchers should begin throwing off the mound by month 9 and can throw up to 180 feet, pain free. Rehabilitation exercises are still 
continued up through this phase concentrating on strength. ${ }^{6}$ The pitcher may increase the pitches to half speed for 25-30 minutes. ${ }^{6}$

By month 9-10, pitchers should still continue to throw at $70 \%-75 \%$ of maximum velocity. ${ }^{6,9}$ It is during this time when the pitcher is able to begin working on a full wind up and this is when biomechanics are suggested to be corrected. ${ }^{9}$ The throwing sessions may also increase from 10-15 minutes up to 25 minute sessions. Some pitchers may also be cleared to return to pitching during this month. ${ }^{22}$

By month 12 , the pitcher may be allowed to return to pitching in competition. ${ }^{9}$ However, before this clearance occurs, the pitcher must meet a certain return to play criteria. This criteria is no pain experienced while throwing, normal strength and ROM in forearm, wrist, elbow and glenohumeral joint, and able to maintain adequate balance through the throwing phase. ${ }^{6,8,9}$ When a pitcher is cleared, pitching 3 innings per game every 7 days is allowed. ${ }^{9}$

As noted throughout the rehabilitation process, different physicians follow different protocols on when an athlete should progress through the phases. Most discrepancies are between phases one and two and when an interval-throwing program can begin and when throws can progress. Overall, most physicians have the same goal throughout a protocol and that is to regain normal ROM and strength and to adequately progress an athlete back to throwing with little to no pain. Once cleared, allowing the athlete to return to competition is the number one goal.

Return To Play

Returning an athlete to practice and competition varies similar to progressions in rehabilitation. An athlete returning to play not only depends on successfully completing rehabilitation, but also on the success of surgical technique used. 
Table B3. Jobe Technique Rehabilitation

\begin{tabular}{|c|c|c|c|c|c|}
\hline Authors & Rehab Protocol & Phase One & Phase Two & Phase Three & Phase Four \\
\hline Azar et al. ${ }^{13}$ & $\begin{array}{l}\text { - } \text { Phase one- Day one } \\
\text { post-op- week (wk) } 3 \\
\text { - Phase two- wk 4-8 } \\
\text { - Phase three- wk 9-13 } \\
\text { - Phase four- wk 14-26 }\end{array}$ & $\begin{array}{l}\text { - Splint- } 90^{\circ}-1 \mathrm{wk} \\
\text { - Hand ROM/wrist } \\
\text { ROM/grip } \\
\text { - Day } 8-\text { ROM } \\
\text { allowed } 30^{\circ}-100^{\circ} \\
\text { - Wk } 3-\text { ROM allowed } \\
15^{\circ}-110^{\circ} \\
\text { - Increase ROM every } \\
\text { wk by } 5^{\circ} \text { extension } \\
\text { and } 10^{\circ} \text { flexion }\end{array}$ & $\begin{array}{l}\text { - End of wk 6- full } \\
\text { ROM } \\
\text { - Isometric strength } \\
\text { exercises } \\
\text { - Progress to isotonic } \\
\text { resistive w/ weight }\end{array}$ & $\begin{array}{l}\text { - Sport-specific exercises } \\
\text { - Proprioceptive } \\
\text { neuromuscular } \\
\text { facilitation (PNF) } \\
\text { - Dynamic stabilization } \\
\text { - Wk 12-plyometric } \\
\text { exercises begin to } \\
\text { prepare for throwing }\end{array}$ & $\begin{array}{l}\text { - Interval throwing program } \\
\text { initiated (wk 14/15) } \\
\text { - Throw } 45 \text { feet, progress to } 180 \\
\text { feet } \\
\text { - Three days a wk } \\
\text { - Pitchers throw long toss at } 120 \\
\text { feet }\end{array}$ \\
\hline Bennett et al. ${ }^{14}$ & $\begin{array}{l}\text { - No timeline stated for } \\
\text { phase progression }\end{array}$ & $\begin{array}{l}\text { - Splint } 90^{\circ}-10 \text { days } \\
\text { - } 30-90 \text { ROM }\end{array}$ & $\begin{array}{l}\text { - Wk 4- progress } \\
\text { ROM 15-105 } \\
\text { - Wk 6- ROM restored } \\
\text { - Strengthening } \\
\text { exercises begin for } \\
\text { glenohumeral joint, } \\
\text { rotator cuff, forearm, } \\
\text { core and lower } \\
\text { extremity }\end{array}$ & $\begin{array}{l}\text { - Wk 12- progress } \\
\text { strengthening exercise } \\
\text { and add weights }\end{array}$ & $\begin{array}{l}\text { - Month 4- interval throwing } \\
\text { program initiated with short } \\
\text { tosses } \\
\text { - Month } 8 \text { - throwing off the } \\
\text { mound begins } \\
\text { - RTP- } 12 \text { months post-op }\end{array}$ \\
\hline Cain et al. ${ }^{10}$ & $\begin{array}{l}\text { - Phase one- Day on post- } \\
\text { op- wk } 3 \\
\text { - Phase two- wk } 4-10 \\
\text { - Phase three- wk } 10-16 \\
\text { - Phase four- wk 16-RTP }\end{array}$ & - Splint- $90^{\circ}-1 \mathrm{wk}$ & $\begin{array}{l}\text { - Wk5/6- full ROM } \\
\text { - Isotonic } \\
\text { strengthening begins } \\
\text { - Throwers Ten } \\
\text { Program }\end{array}$ & $\begin{array}{l}\text { - Sport-specific } \\
\text { - Wk 12- isotonic lifting } \\
\text { begins and two-hand } \\
\text { plyometric exercises } \\
\text { - Wk 14- One handed } \\
\text { plyometric exercises } \\
\text { - Core and leg } \\
\text { strengthening begins }\end{array}$ & $\begin{array}{l}\text { - Interval throwing program } \\
\text { initiated }\end{array}$ \\
\hline Conway et al. ${ }^{9}$ & $\begin{array}{l}\text { - No timeline stated for } \\
\text { phase progression }\end{array}$ & - Splint $90^{\circ}-10$ days & $\begin{array}{l}\text { - Wk 4/6- } \\
\text { wrist/forearm } \\
\text { strengthening } \\
\text { exercises begin } \\
\text { - Wk7- Elbow } \\
\text { strengthening begins, } \\
\text { avoiding valgus }\end{array}$ & $\begin{array}{l}\text { - Sport-specific } \\
\text { exercises }\end{array}$ & $\begin{array}{l}\text { - Month 4- interval throwing } \\
\text { program initiated } \\
\text { - } 30-40 \text { feet toss to begin. 2-3 } \\
\text { x's/wk. 10-15 minutes total } \\
\text { - Month 5- } 60 \text { feet toss } \\
\text { - Month 6- Lobs toss to } 60 \text { feet } \\
\text { w/ easy windup. }\end{array}$ \\
\hline
\end{tabular}


stress

\begin{tabular}{|c|c|c|}
\hline Jobe et al. $^{8}$ & $\begin{array}{l}\text { - No timeline stated for } \\
\text { phase progression }\end{array}$ & $\begin{array}{l}\text { - Splint } 90-2 \text { wks } \\
\text { - Ball squeezes begin } \\
\text { - Wk 2- elbow and } \\
\text { glenohumeral joint } \\
\text { ROM exercises } \\
\text { begin }\end{array}$ \\
\hline Patel et al. ${ }^{4}$ & $\begin{array}{l}\text { - Phase one- wk } 1 \\
\text { - Phase two- wk 2-4 } \\
\text { - Phase three- wk 6-10 } \\
\text { - Phase four- wk 11-RTP }\end{array}$ & $\begin{array}{l}\text { - Splint- } 90^{\circ} \\
\text { - Wrist ROM and } \\
\text { grip exercises } \\
\text { begin } \\
\text { - Isometric exercises } \\
\text { of wrist, hand and } \\
\text { elbow }\end{array}$ \\
\hline
\end{tabular}

- Month 1- increase muscle

strengthening, progress to light weights

- Begin glenohumeral joint and rotator cuff strengthening

- ROM progress $40^{\circ}$ $100^{\circ}$

- Wk 5/6- full ROM restored

- Isometric exercises progressed

- Introduce isotonic exercises- Scapular stabilizations/gleno humeral joint exercises

- Avoid valgus loads/stresses

- Upper body PNF introduced
- Month 7- half speed throwing from mound. 25 minutes

- Month 8-9- 75\% max pitching velocity

- Month 10-Continue 75\% throwing velocity, work on wind up and biomechanics

- Month 12- Competitive pitching

- Throwing program was not stated, but does mention to stop throwing if pain or swelling occurs for 2 wks.

- Shoulder external rotation allowed

- Wk 16- interval throwing program begins

- Continue

glenohumeral joint,

forearm, elbow and wrist exercises

- Wk 9/10- eccentric loads allowed

- Plyometric exercises introduced

- Sport-specific drills

- Functional training for lower extremity/core 
Table B4. Docking Technique Rehabilitation

\begin{tabular}{|c|c|c|c|c|c|}
\hline Authors & Rehab Protocol & Phase One & Phase Two & Phase Three & Phase Four \\
\hline Bowers et al. $^{20}$ & $\begin{array}{l}\text { - No timeline stated } \\
\text { for phase } \\
\text { progression }\end{array}$ & $\begin{array}{l}\text { - Wk 1- splint } 90^{\circ} \\
\text { - } 30^{\circ}-90^{\circ} \text { ROM }\end{array}$ & $\begin{array}{l}\text { - Wk } 4-15^{\circ}-105^{\circ} \\
\text { ROM } \\
\text { - Wk 6- Full ROM \& } \\
\text { brace discontinued }\end{array}$ & & $\begin{array}{l}\text { - } 4 \text { months-interval throwing program } \\
\text { begins } \\
\text { - } 8 \text { months- off the mound throwing }\end{array}$ \\
\hline Jones et al. ${ }^{18}$ & $\begin{array}{l}\text { - No timeline stated } \\
\text { for phase } \\
\text { progression }\end{array}$ & $\begin{array}{l}\text { - } 10 \text { days- splint } \\
50^{\circ}-60^{\circ} \text { flexion } \\
\text { - Wk } 1-30^{\circ}-90^{\circ} \\
\text { ROM }\end{array}$ & $\begin{array}{l}\text { - Wk4/6- Full ROM } \\
\text { restored }\end{array}$ & & $\begin{array}{l}\text { - Month 4- plyometric exercises begin } \\
\text { and interval throwing program begins } \\
\text { - Month 4- Core/lower extremity } \\
\text { strengthen begins }\end{array}$ \\
\hline Dodson et al. ${ }^{21}$ & $\begin{array}{l}\text { - No timeline stated } \\
\text { for phase } \\
\text { progression } \\
\text { - Physical therapy } \\
\text { does not start until } \\
\text { week } 6\end{array}$ & $\begin{array}{l}\text { - Wk 1-splint } 90^{\circ} \\
\text { - Wk 2- } 30^{\circ}-90^{\circ} \\
\text { ROM } \\
\text { - Wk 3-5- } 15^{\circ}- \\
105^{\circ} \text { ROM }\end{array}$ & $\begin{array}{l}\text { - Wk 6- PT begins } \\
\text { - Rotator cuff, } \\
\text { glenohumeral joint } \\
\text { and forearm } \\
\text { strengthening } \\
\text { begins }\end{array}$ & $\begin{array}{l}\text { - Wk 12- plyometric } \\
\text { exercises begin } \\
\text { - Core and trunk } \\
\text { exercises begin } \\
\text { - Progress } \\
\text { glenohumeral joint } \\
\text { and rotator cuff } \\
\text { exercises }\end{array}$ & $\begin{array}{l}\text { Month 4-Interval throwing begins- } 45 \\
\text { feet toss, every other day. Progress } \\
\text { when pain free } \\
\text { Month 9- off the mound throwing- up } \\
180 \text { feet }\end{array}$ \\
\hline Meyers et al. ${ }^{3}$ & $\begin{array}{l}\text { - Early ROM is key } \\
\text { - Phase one- wk 1-2 } \\
\text { - Week } 3-15^{\circ}-110^{\circ} \\
\text { ROM } \\
\text { - Phase two- wk 4-8 } \\
\text { - Phase three- wk 9- } \\
36 \\
\text { - Phase four- 9-12 } \\
\text { months }\end{array}$ & $\begin{array}{l}\text { - } 10 \text { days- splint } \\
90^{\circ} \\
\text { - Wrist/hand } \\
\text { ROM and grip } \\
\text { exercises } \\
\text { - } 30^{\circ}-100^{\circ} \mathrm{ROM} \\
\text { - } \text { Isometric } \\
\text { exercises }\end{array}$ & $\begin{array}{l}\text { - Progress ROM by } \\
5^{\circ} \text { extension and } \\
10^{\circ} \text { flexion each } \\
\text { week } \\
\text { - Progress isometric } \\
\text { exercises } \\
\text { - Full ROM restored }\end{array}$ & $\begin{array}{l}\text { - Sport specific } \\
\text { exercises } \\
\text { - Wk 16- Throwing } \\
\text { program initiated } \\
\text { - } 45 \text { feet-120 feet } \\
\text { - Pitchers progress } \\
\text { from half velocity, } \\
\text { three-quarter } \\
\text { velocity, to full } \\
\text { velocity }\end{array}$ & $\begin{array}{l}\text { - Progress to returning to competition } \\
\text { during this phase }\end{array}$ \\
\hline Patel et al. ${ }^{4}$ & $\begin{array}{l}\text { - Phase one- wk } 1 \\
\text { - Phase two- wk 2-4 } \\
\text { - Phase three- wk 6- } \\
10 \\
\text { - Phase four- wk 11- } \\
\text { RTP }\end{array}$ & $\begin{array}{l}\text { - Splint- } 90^{\circ} \\
\text { - Wrist ROM and } \\
\text { grip exercises } \\
\text { begin } \\
\text { - Isometric } \\
\text { exercises of } \\
\text { wrist, hand and } \\
\text { elbow }\end{array}$ & $\begin{array}{l}\text { - } \mathrm{ROM} \text { progress } 40^{\circ}- \\
100^{\circ} \\
\text { - } \mathrm{Wk} 5 / 6-\text { full ROM } \\
\text { restored } \\
\text { - Isometric exercises } \\
\text { progressed } \\
\text { - Introduce isotonic } \\
\text { exercises- Scapular }\end{array}$ & $\begin{array}{l}\text { - Glenohumeral joint } \\
\text { external rotation } \\
\text { allowed } \\
\text { - Continue } \\
\text { glenohumeral } \\
\text { joint, forearm, } \\
\text { elbow and wrist } \\
\text { exercises }\end{array}$ & $\begin{array}{l}\text { - Wk 16- interval throwing program } \\
\text { begins }\end{array}$ \\
\hline
\end{tabular}




\begin{tabular}{|c|c|c|c|c|c|}
\hline Rohrbough et al 22 & & & $\begin{array}{l}\text { stabilizations/gleno } \\
\text { humeral joint } \\
\text { exercises } \\
\text { - Avoid valgus } \\
\text { loads/stresses } \\
\text { - Upper body PNF } \\
\text { introduced }\end{array}$ & $\begin{array}{l}\text { - Wk 9/10- eccentric } \\
\text { loads allowed } \\
\text { - Plyometric } \\
\text { exercises } \\
\text { introduced } \\
\text { - Sport-specific drills } \\
\text { - Functional training } \\
\text { for lower } \\
\text { extremity/core }\end{array}$ & \\
\hline
\end{tabular}


The literature reports multiple different time frames in which baseball players' return to competitive pitching. Overall, most studies will report the athletes returning within 12 months, ${ }^{3,6,8,10,13,18,20,21}$ or within 18 months. ${ }^{9}$ When it comes to returning specific levels of baseball athletes to play, only one study suggested professional baseball players returning within 12 months. ${ }^{13}$ However, multiple studies suggest that professional baseball players return to play within 16.8 months, ${ }^{41} 18$ months, ${ }^{6,42}$ and 20 months. ${ }^{43}$ Only one study reported specifically about collegiate players who are allowed to return within 12 months. ${ }^{6}$

Comparing the success of the Docking technique to the Jobe technique, a $68 \%$ return rate for the Jobe technique ${ }^{9}$ and a $92 \%$ return rate for the Docking technique were noted. ${ }^{22}$ About 73 $75 \%$ of all baseball players in the studies will return to their previous playing level. ${ }^{10,13}$ One study reported a $97 \%$ success rate for professional baseball players returning to MLB. ${ }^{43}$ Collegiate baseball players return to their previous level of competition only $67 \%$ of the time, when the Jobe technique was used. ${ }^{10}$ It is reported in one study that $>50 \%$ of pitchers who undergo UCL reconstruction will end up returning to the disabled list, and missing games due to throwing arm injuries. ${ }^{41}$ Twenty-six percent of pitchers will return to the disabled list specifically for elbow re-injury. ${ }^{41}$

When a significant number of athletes have a good success rate to return to play, there was a decrease in pitching statistics. It is found that when an athlete returns to pitching in a game, the earned run average (ERA) and walk plus hit per inning pitch (WHIP) increases. ${ }^{41,43}$ It was also found that these pitchers who underwent UCL reconstruction, pitched in fewer games, and when they did pitch, the pitch count was drastically lower. ${ }^{41,43}$ It was also reported that there was a significant drop in percent of pitches within the strike zone and the number of fastballs thrown. $^{41}$ 
Aside from a drop in statistics for the pitchers there was also a drop in the workload. It is reported that relief pitchers were able to return to throwing $50 \%$ of pre-injury level. ${ }^{42}$ For starting pitchers, rates are lower than relief pitchers. The starting pitchers were only able to return to $35 \%$ of pre-injury level. ${ }^{42}$

\section{SUMMARY}

Overall, UCL injuries may not be as prevalent as glenohumeral injuries in baseball, but they are just as debilitating to an athlete when they are sustained. When a UCL injury occurs, it is typically due to micro trauma to the elbow, causing overuse injuries. There is still a chance that a UCL can tear in an acute incidence, but this is not as common. The UCL is composed of 3 ligaments: anterior bundle, posterior band and transverse (oblique/coopers) ligament. The anterior bundle is composed of 2 bands: the anterior and posterior bands. It is the anterior bundle that is most commonly torn/injured when a UCL injury occurs.

The treatment for UCL injuries is an extensive process that can last anywhere from 3-20 months, depending on the route taken. There is a chance that a non-surgical option can be explored and the athlete can return to play with no surgery, where in most cases, surgery is deemed necessary. There are different surgical techniques that can be used to reconstruct the UCL, but the two more common techniques are the Jobe and Docking techniques. The main difference between these techniques is the number of tunnels drilled, ulnar nerve transposition, and the form of the graft ("figure-8" vs. "Y-shaped"). The success rate for these two techniques is different. The Jobe technique has a return to play rate of $68 \%$, while the Docking technique's rate is $92 \%$. Since the evolution of the Docking technique, more athletes have been able to return to play baseball more so than following the Jobe technique, leading to the Docking technique being more successful. 
There is controversy as to when an athlete can progress from phase one to phase two and when full ROM should be restored. There also is a discrepancy on when an interval-throwing progression should start. Some authors' state it is safe for throwing to begin after 14 weeks postop, while others feel 4 months is better to begin. Even with this 2-week difference, an athlete's return to play rate or a delay in returning based on pain can be impacted.

When it comes to returning an athlete to play, they must successfully complete the interval-throwing progression ending with throwing off the mound at $100 \%$ velocity. If the athlete can throw pain free, then a return to game progression begins by allowing 3 innings every 7 days. Some pitchers statistics are changed due to UCL reconstruction by an increase in ERA and WHIP. 


\section{APPENDIX C}

\section{ADDITIONAL METHODS}

Table C1. Inclusion/Exclusion Criteria

1. The studies will be written in English or an English translation.

2. Ulnar collateral ligament, baseball athletes, throwing athletes, surgical techniques, elbow, reconstruction, rehabilitation, or various forms of theses terms will be mentioned in the title of the study.

3. Ulnar collateral ligament, baseball athletes, throwing athletes, surgical techniques, elbow, reconstruction, rehabilitation, or various forms of theses terms will be mentioned in the abstract.

4. Ulnar Collateral ligament reconstruction and rehabilitation will be the main focus of the study.

5. The study will be an experimental study or cohort study. 
Table C2. Coleman Methodology Score (CMS) ${ }^{24}$

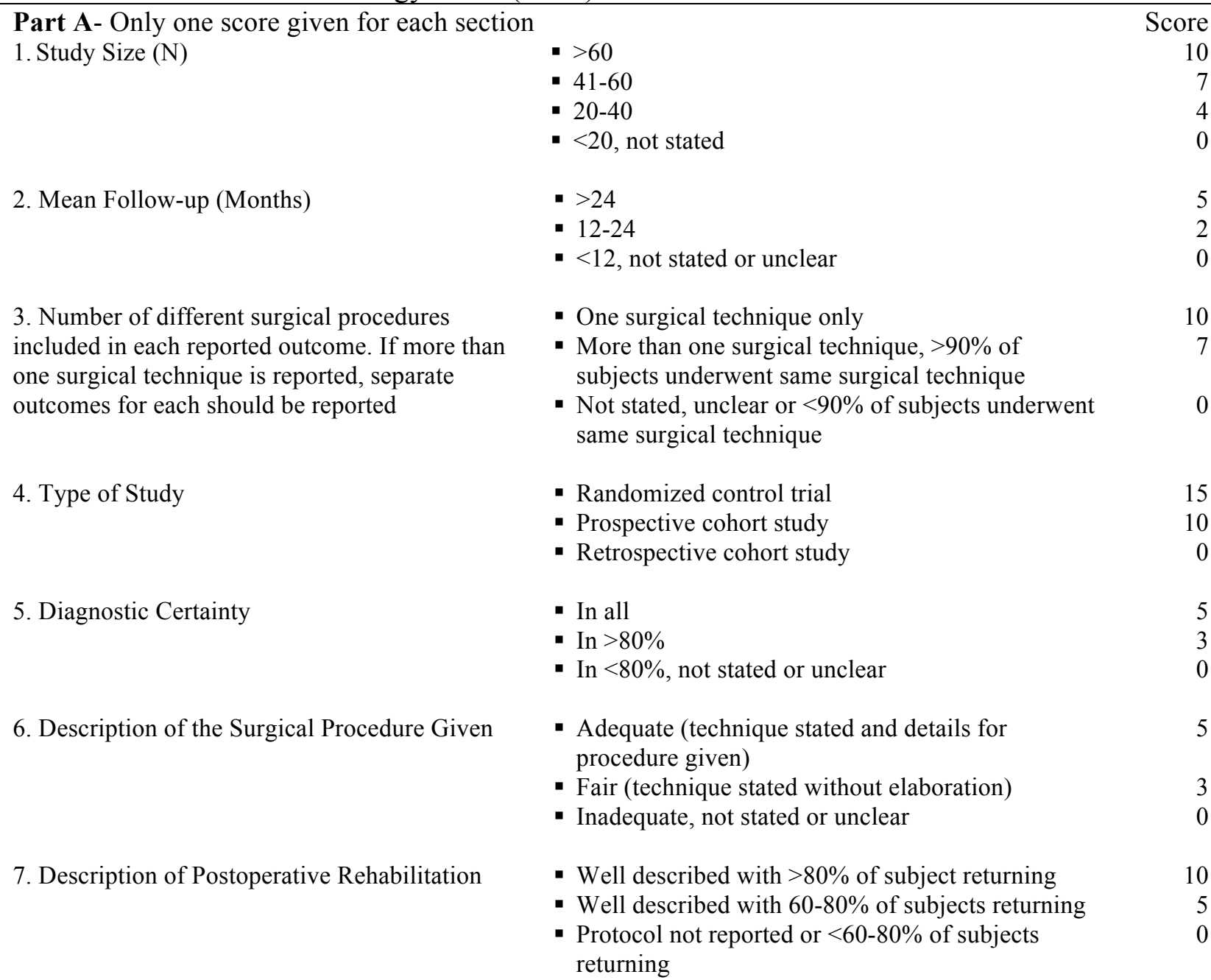

Part B- Score may be given for each option in all three sections

8. Outcome Criteria

(If outcome criteria is vague, score is

automatically 0 for section)

9. Procedure for Assessing Outcomes

10. Description of Subject Selection Process
- Outcome measures clearly defined 2

- Timing of outcome assessment clearly stated 2

- Use of outcome criteria that has reported good 3 reliability

- Use of outcome with good sensitivity 3

- Subjects recruited (not taken from surgeons' files) 5

- Investigator independent of surgeon 4

- Written assessment 3

- Completion of assessment by subjects themselves 3 with minimal investigator assistance

- Selection criteria reported and unbiased 5

- Recruitment rate reported- $>80 \%$ or 5 $<80 \%$

- Eligible subjects not included in study 5 satisfactorily accounted for or $100 \%$ recruitment

Total: $/ 100$ 
Table C3. Descriptions of Coleman Methodology Score (CMS)

Part A. One score is given for each section

Section 1. Study Size (N)

Explanation: When choosing the score for this section, if the study states the study size is $>60$ subjects, the appropriate score is 10 . When the study size falls between $41-60$, the score given should be 7 . If the study size falls between $20-40$, the score given should be 4 . If the study size is less than 20 subjects or is not stated, the score given should be a 0 . If there were multiple followups, multiply the study size number by the number of follow-ups for the subjects points possible.

Section 2. Mean Follow-up (Months)

Explanation: When a follow-up the frame is stated (either in the methods section, results of discussion), a score of 5 is given when the time frame is $>24$ months. When the follow-up times fall between 12-24 months, a score of 2 is given. When the follow-up is $<12$ months, not stated or is unclear, a score of 0 is given points possible.

Section 3. Number of different surgical procedures included in each reported outcome. If more than one surgical technique is reported, separate outcomes for each technique used should be reported.

Explanation: In the Methods or Surgical Treatment sections of studies, when a surgical intervention is mentioned, if only one surgical technique is used for $100 \%$ of the subjects, a score of 10 is given. When one technique is used on $>90 \%$ of the subjects, a score of 7 is to be given. When the number of subjects who underwent the same surgical technique is $<90 \%$, the number of subjects within different surgical techniques is not stated or unclear, a score of 0 is given. If there are multiple surgical techniques used within the study, this criterion will be repeated for each surgical technique reported points possible.

Section 4. Type of Study

Explanation: Mentioned in the studies abstract (as level or type of evidence) or within the Methods section of the study, if the study is considered to be a randomized control study, a score of 15 is to be given. When it is stated that the study is a prospective cohort study, a score of 10 is to be given. If the study states it is a retrospective cohort study, a score of 0 is to be given points possible.

\section{Section 5. Diagnostic Certainty}

Explanation: If the use of preoperative ultrasound, magnetic resonance imaging (MRI), or postop histopathology is used to diagnosis the injury in all the subjects in the study, a score of 5 is given. If the use of preoperative diagnostic tools is used in $>80 \%$ of the subjects within the study, a score of 3 is given. If $<80 \%$ of the subjects received a positive diagnostic test to diagnosis the injury, it is unclear or not stated, a score of 0 is given points possible.

Section 6. Description of the Surgical Procedure Given Explanation: In the Methods or Procedure section(s), when the researcher explains the surgical techniques procedure, if the procedure is described in adequate amount of detail, meaning that the technique is stated and specific details about the procedure are given, a score of 5 is given. A fair score is chosen when the technique is stated but the researchers do not go into much detail 
about the specific technique, a score of 3 is given. If the surgical technique is not stated, inadequate or unclear, a score a 0 is given point possibility.

Section 7. Description of Postoperative Rehabilitation

Explanation: Near the end of the surgical technique used, in a rehabilitation section, or found in the results section, if the rehabilitation involved with the technique goes into great amount of detail with the phases of rehabilitation and $>80 \%$ of the subjects return to sport, a score of 10 is given. If the rehabilitation is described well, but only $60-80 \%$ of the subject return, a score of 5 is given. When the protocol is not reported at all or $<60 \%$ of the subjects return to the sport, a score of 0 is given point possibility.

Part B. Multiple scores may be given within each section, as the study meets the criterion within each section.

Section 8. Outcome Criteria

Explanation: If the criterion is vague in the study, a score of 0 is automatically given. If the studies outcome measures are all clearly defined, a score of 2 is given. A score of 2 is also given if the timing of the outcome assessments is clearly defined/stated. A score of 3 is given when the reliability is reported as good. A score of 3 is also given if the sensitivity of the study is good. In this section, the point possibility is a minimum of 0 to a maximum of 10 .

Section 9. Procedure for Assessing Outcomes

Explanation: If the study reported the subjects were recruited, a score of 5 is given. If the investigator is independent of the surgeon, meaning that the publishing authors are not the surgeons performing the surgery, a score of 4 is given. If there is a written assessment used on the subjects but filled out by the investigator, a score of 3 is given. If there is a written assessment given to the subjects to fill out independently, a score of 3 is given. In this section, the point possibility is a minimum of 3 to a maximum of 15 points.

Section 10. Description of Selection Process

Explanation: If the selection criterion for the study is clearly reported and unbiased, a score of 5 is given. In the chance the subjects for the study are recruited, if the reported recruitment rate is $>80 \%$, a score of 5 is given. But if $<80 \%$ of the subjects are recruited, a score of 3 is given. In the inclusion/exclusion criteria for the study, if the eligibility of the subjects is not included or there is a $100 \%$ recruitment rate, a score of 5 is given. 


\section{APPENDIX D}

\section{ADDITIONAL RESULTS}

Table D1. Jobe Surgical Technique Studies

\begin{tabular}{|c|c|c|c|c|c|}
\hline Study & Patients & Interventions & Outcomes & Conclusion & CMS \\
\hline Azar et al. ${ }^{13}$ & $\begin{array}{l}\text { - } 78 \text { males w/ UCL } \\
\text { reconstruction } \\
\text { - } 13 \text { males w/ UCL repair }\end{array}$ & $\begin{array}{l}\text { - Jobe technique } \\
\text { - } 2 \text { modifications: } \\
\text { flexor pronator } \\
\text { mass not detached } \\
\text { and ulnar nerve } \\
\text { transposition }\end{array}$ & - Refer to Table B1 & - Refer to Table B1 & 39 \\
\hline Cain et al. ${ }^{10}$ & $\begin{array}{l}\text { - } 1266 \text { UCL reconstruction } \\
\text { - } 15 \text { UCL repair } \\
\text { - } 253 \text { males } \\
\text { - } 28 \text { females }\end{array}$ & $\begin{array}{l}\text { - Jobe technique } \\
\text { - } 2 \text { modifications: } \\
\text { flexor pronator } \\
\text { mass not detached } \\
\text { and ulnar nerve } \\
\text { transposition }\end{array}$ & - Refer to Table B1 & - Refer to Table B1 & 50 \\
\hline Dugas et al. $^{26}$ & $\begin{array}{l}\text { - } 120 \text { patients } \\
\text { - } 42 \text { placed in bony group } \\
\text { - } 78 \text { placed in nonbony } \\
\text { group }\end{array}$ & $\begin{array}{l}\text { - Jobe technique } \\
\text { - } 2 \text { modifications: } \\
\text { flexor pronator } \\
\text { mass not detached } \\
\text { and ulnar nerve } \\
\text { transposition }\end{array}$ & $\begin{array}{l}\text { - } 34 \text { patients classified as excellent for } \\
\text { bony group in RTP } \\
\text { - } 71 \text { patients classified as excellent for } \\
\text { nonbony group in RTP } \\
\text { - } 3 \text { patients classified as good for bony } \\
\text { group in RTP } \\
\text { - } 1 \text { patient in both bony and nonbony } \\
\text { groups classified as fair } \\
\text { - } 4 \text { patients classified as poor in bony } \\
\text { group } \\
\text { - } 6 \text { patients classified as poor in bony } \\
\text { group } \\
\text { - All classifications are based on } \\
\text { Conway-Jobe scale }\end{array}$ & $\begin{array}{l}\text { - } 89 \% \text { RTP with use of gracilis } \\
\text { tendon graft } \\
\text { - Those in the nonbony group } \\
(91 \%) \text { were more successful in } \\
\text { RTP over bony group }(81 \%) \\
\text { - Decrease in pitch control noted } \\
\text { for bony group }\end{array}$ & 62 \\
\hline Jobe et al. $^{8}$ & - 16 male throwing athletes & - Jobe technique & - Refer to Table B1 & - Refer to Table B1 & 37 \\
\hline Merolla et al. ${ }^{27}$ & - 26 patients under went & - Jobe technique & - 10 patients classified as excellent for & - No difference was found for & 47 \\
\hline
\end{tabular}


UCL surgery

- 15 used in study

- 5 used Jobe technique

Osbahr et al. ${ }^{28} \quad$ - 313 patients total

- 256 used in study
RTP

- 4 patients classified as good for RTP

- 1 patient classified as poor for RTP

- All classifications are based on

Conway-Jobe scale

- MEPS, OES and DASH scores were $(p<0.01)$

- More surgeries used the palmaris graft over semitendiosus $(p>0.05)$

- Jobe technique

- 2 modifications: flexor pronator mass not detached and ulnar nerve transposition
- After a minimum of a 10 year follow patients)

- 13 still remain active in sport $(5 \%$ patients)

- $83 \%$ returned to same level or higher of competition up, 243 retired from baseball (95\% outcomes when comparing allograft to autograft

- Most difficult aspect of the surgical procedure was fitting the graft used through the narrow tunnels. Surgeons reported wanting to avoid widening the tunnels to avoid weakening the bone

- 6 athletes returned to previous level of competitions between 610 months

- $93 \%$ satisfaction reported from all players

- Few reported pain after surgery

- Retirement was not due to elbow

- Excellent results reported for disability and outcome scales

Table D2. Docking Surgical Technique Studies

\begin{tabular}{|c|c|c|c|c|c|}
\hline Study & Patients & Interventions & Outcomes & Conclusion & CMS \\
\hline Bowers et al. $^{20}$ & $\begin{array}{l}\text { - } 21 \text { overhead-throwing } \\
\text { athletes }\end{array}$ & $\begin{array}{l}\text { - Docking } \\
\text { technique } \\
\text { - Excessive graft } \\
\text { sew to limb of } \\
\text { graft }\end{array}$ & - Refer to Table B2 & - Refer to Table B2 & 46 \\
\hline Dodson et al. ${ }^{21}$ & $\begin{array}{l}100 \text { male overhead- } \\
\text { throwing athletes }\end{array}$ & $\begin{array}{l}\text { - Docking } \\
\text { technique }\end{array}$ & - Refer to Table B2 & - Refer to Table B2 & 62 \\
\hline Koh et al. ${ }^{19}$ & $\begin{array}{l}\text { - } 20 \text { high-level baseball } \\
\text { players }\end{array}$ & $\begin{array}{l}\text { - Docking } \\
\text { technique } \\
\text { - Two strand and } \\
\text { three strand graft } \\
\text { used }\end{array}$ & $\begin{array}{l}\text { - } 18 \text { RTP to same level of competition or } \\
\text { higher } \\
\text { - } 2 \text { RTP changed positions due to not } \\
\text { wanting to pitch } \\
\text { - } 17 \text { patients classified as excellent for } \\
\text { RTP }\end{array}$ & - Technique reliable for RTP & 51 \\
\hline
\end{tabular}




\begin{tabular}{|c|c|c|c|c|c|}
\hline Merolla et al. ${ }^{27}$ & $\begin{array}{l}\text { - } 26 \text { patients under went } \\
\text { UCL surgery } \\
\text { - } 15 \text { used in study } \\
\text { - } 8 \text { used Docking } \\
\text { technique }\end{array}$ & $\begin{array}{l}\text { - Docking } \\
\text { technique }\end{array}$ & - Refer to Table D1 & - Refer to Table D1 & 47 \\
\hline Paletta et al. ${ }^{29}$ & - 25 baseball players & $\begin{array}{l}\text { - Docking } \\
\text { technique }\end{array}$ & $\begin{array}{l}\text { - } 23 \text { RTP to same level of competition or } \\
\text { higher }\end{array}$ & - $92 \%$ of athletes RTP & 46 \\
\hline Rohrbough et al. ${ }^{22}$ & $\begin{array}{l}\text { - } 36 \text { patients } \\
\text { - } 35 \text { male patients } \\
\text { - } 1 \text { female patient }\end{array}$ & $\begin{array}{l}\text { - Docking } \\
\text { technique }\end{array}$ & - Refer to Table B2 & - Refer to Table B2 & 51 \\
\hline Savoie et al..$^{30}$ & $\begin{array}{l}\text { - } 123 \text { overhead- } \\
\text { throwing athletes } \\
\text { - } 116 \text { used in study }\end{array}$ & $\begin{array}{l}\text { - Docking } \\
\text { technique }\end{array}$ & $\begin{array}{l}\text { - } 6 \text { patients did not RTP } \\
\text { - } 33 \text { patients RTP to higher levels of } \\
\text { competition } \\
\text { - } 64 \text { patients RTP to previous levels of } \\
\text { competition } \\
\text { - } 13 \text { RTP below previous level of } \\
\text { competition } \\
\text { - } 93 \text { patients classified as excellent for } \\
\text { RTP } \\
\text { - } 15 \text { patients classified as good for RTP } \\
\text { - } 8 \text { patients classified as fair for RTP } \\
\text { - All classifications based on Conway- } \\
\text { Jobe scores }\end{array}$ & $\begin{array}{l}\text { - Advantage of allograft is } \\
\text { decrease in surgery time } \\
\text { - } 88 \% \text { RTP to same level or higher }\end{array}$ & 52 \\
\hline
\end{tabular}

- 2 patients classified as good for RTP

- All classifications are based on

Conway-Jobe scale

23 RTP to same level of competition or $\quad$ - $92 \%$ of athletes RTP

35 male patients

123 overhead-

throwing athletes

6 patients did not RTP

competition

Advantage of allograft is

64 patients RTP to previous levels of

competition

$$
\text { RTP }
$$

Jobe scores 
Table D3. Coleman Methodology Score (CMS) Results

\begin{tabular}{|c|c|c|c|c|c|c|c|c|c|c|c|}
\hline Study & Study Size & Follow-up & $\begin{array}{l}\text { Number } \\
\text { Procedures }\end{array}$ & $\begin{array}{l}\text { Type } \\
\text { of } \\
\text { Study }\end{array}$ & Diagnostic & Procedure & Rehab & Outcomes & $\begin{array}{l}\text { Assessing } \\
\text { Outcomes }\end{array}$ & $\begin{array}{c}\text { Subject } \\
\text { Selection }\end{array}$ & $\begin{array}{l}\text { Total } \\
\text { Score }\end{array}$ \\
\hline Azar et al. ${ }^{13}$ & 10 & 5 & 7 & 0 & 0 & 5 & 5 & 2 & 0 & 5 & 39 \\
\hline Bowers et al..$^{20}$ & 4 & 5 & 10 & 0 & 0 & 5 & 10 & 7 & 0 & 5 & 46 \\
\hline Cain et al. ${ }^{10}$ & 10 & 5 & 10 & 0 & 5 & 3 & 10 & 2 & 0 & 5 & 50 \\
\hline Dodson et al..$^{21}$ & 10 & 5 & 10 & 0 & 5 & 5 & 10 & 7 & 0 & 10 & 62 \\
\hline Dugas et al. ${ }^{26}$ & 10 & 5 & 10 & 0 & 5 & 5 & 10 & 7 & 0 & 10 & 62 \\
\hline Jobe et al. ${ }^{8}$ & 0 & 5 & 10 & 0 & 0 & 5 & 5 & 4 & 3 & 5 & 37 \\
\hline Koh et al. ${ }^{19}$ & 4 & 5 & 7 & 0 & 5 & 5 & 10 & 7 & 3 & 5 & 51 \\
\hline Merolla et al. ${ }^{27}$ & 4 & 5 & 0 & 0 & 5 & 3 & 5 & 10 & 10 & 5 & 47 \\
\hline Osbahr et al. ${ }^{28}$ & 10 & 5 & 10 & 0 & 0 & 3 & 10 & 7 & 6 & 5 & 47 \\
\hline Paletta et al. ${ }^{29}$ & 4 & 5 & 10 & 0 & 5 & 5 & 10 & 2 & 0 & 5 & 46 \\
\hline Rohrbough et al. ${ }^{22}$ & 4 & 5 & 10 & 0 & 5 & 5 & 10 & 7 & 0 & 5 & 51 \\
\hline Savoie et al. ${ }^{30}$ & 10 & 5 & 7 & 0 & 5 & 3 & 5 & 7 & 0 & 10 & 52 \\
\hline
\end{tabular}


Table D4. Azar et al. ${ }^{13}$ Coleman Methodology Score

\begin{tabular}{|c|c|}
\hline Part A- Only one score given for each section & \\
\hline 1.Study Size (N) & - $>60$ \\
\hline & - $41-60$ \\
\hline & - $20-40$ \\
\hline & - $<20$, not stated \\
\hline 2. Mean Follow-up (Months) & - $>24$ \\
\hline & - $12-24$ \\
\hline & - $<12$, not stated or unclear \\
\hline 3. Number of different surgical procedures & - One surgical technique only \\
\hline $\begin{array}{l}\text { included in each reported outcome. If more than } \\
\text { one surgical technique is reported, separate }\end{array}$ & $\begin{array}{l}\text { - More than one surgical technique, }>90 \% \text { of } \\
\text { subjects underwent same surgical technique }\end{array}$ \\
\hline outcomes for each should be reported & $\begin{array}{l}\text { - Not stated, unclear or }<90 \% \text { of subjects underwent } \\
\text { same surgical technique }\end{array}$ \\
\hline 4. Type of Study & - Randomized control trial \\
\hline & $\begin{array}{l}\text { - Prospective cohort study } \\
\text { - Retrospective cohort study }\end{array}$ \\
\hline 5. Diagnostic Certainty & - In all \\
\hline & - In $>80 \%$ \\
\hline & - In $<80 \%$, not stated or unclear \\
\hline 6. Description of the Surgical Procedure Given & $\begin{array}{l}\text { - Adequate (technique stated and details for } \\
\text { procedure given) }\end{array}$ \\
\hline & - Fair (technique stated without elaboration) \\
\hline & - Inadequate, not stated or unclear \\
\hline 7. Description of Postoperative Rehabilitation & - Well described with $>80 \%$ of subject returning \\
\hline & - Well described with $60-80 \%$ of subjects returning \\
\hline & $\begin{array}{l}\text { - Protocol not reported or }<60-80 \% \text { of subjects } \\
\text { returning }\end{array}$ \\
\hline
\end{tabular}

Part B- Score may be given for each option in all three sections

8. Outcome Criteria

(If outcome criteria is vague, score is

automatically 0 for section)

9. Procedure for Assessing Outcomes

10. Description of Subject Selection Process
- Outcome measures clearly defined

- Timing of outcome assessment clearly stated

- Use of outcome criteria that has reported good reliability

- Use of outcome with good sensitivity

- Subjects recruited (not taken from surgeons' files) 5

- Investigator independent of surgeon

- Written assessment

- Completion of assessment by subjects themselves with minimal investigator assistance

- Selection criteria reported and unbiased 5

- Recruitment rate reported- $>80 \%$ or 5 $<80 \%$

- Eligible subjects not included in study satisfactorily accounted for or $100 \%$ recruitment

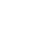


Table D5. Bowers et al. ${ }^{20}$ Coleman Methodology Score

Part A- Only one score given for each section Score 1.Study Size (N)

- $>60$

- 41-60

- $20-40$

- $<20$, not stated

2. Mean Follow-up (Months)

- $>24$

- $12-24$

- $<12$, not stated or unclear

- One surgical technique only

- More than one surgical technique, $>90 \%$ of subjects underwent same surgical technique

3. Number of different surgical procedures
included in each reported outcome. If more than one surgical technique is reported, separate outcomes for each should be reported

- Not stated, unclear or $<90 \%$ of subjects underwent same surgical technique
4. Type of Study

5. Diagnostic Certainty

6. Description of the Surgical Procedure Given

7. Description of Postoperative Rehabilitation
- Randomized control trial

- Prospective cohort study

- Retrospective cohort study

- In all

- In $>80 \%$

- In $<80 \%$, not stated or unclear

- Adequate (technique stated and details for procedure given)

- Fair (technique stated without elaboration)

- Inadequate, not stated or unclear

- Well described with $>80 \%$ of subject returning

- Well described with $60-80 \%$ of subjects returning

- Protocol not reported or $<60-80 \%$ of subjects returning
10

7

$\underline{4}$

0

5

2

0

10

7

0

15

10

0

5

Part B- Score may be given for each option in all three sections

8. Outcome Criteria (If outcome criteria is vague, score is automatically 0 for section)

9. Procedure for Assessing Outcomes

10. Description of Subject Selection Process
- Outcome measures clearly defined

- Timing of outcome assessment clearly stated

- Use of outcome criteria that has reported good reliability

- Use of outcome with good sensitivity

- Subjects recruited (not taken from surgeons' files)

- Investigator independent of surgeon

- Written assessment

- Completion of assessment by subjects themselves with minimal investigator assistance

- Selection criteria reported and unbiased 5

- Recruitment rate reported- $>80 \%$ or 5 $<80 \%$

- Eligible subjects not included in study satisfactorily accounted for or $100 \%$ recruitment $\underline{2}$

$\underline{\mathbf{2}}$

$\underline{3}$

3

5

4

3

3

5
5
3
5

Total: $46 / 100$ 
Table D6. Cain et al. ${ }^{10}$ Coleman Methodology Score

Part A- Only one score given for each section

1.Study Size (N)

- $>60$

Score

- $41-60$

$\underline{10}$

- $20-40$

- $<20$, not stated

- $>24$

- $12-24$

- $<12$, not stated or unclear

3. Number of different surgical procedures

- One surgical technique only included in each reported outcome. If more than one surgical technique is reported, separate outcomes for each should be reported

- More than one surgical technique, $>90 \%$ of subjects underwent same surgical technique

- Not stated, unclear or $<90 \%$ of subjects underwent same surgical technique

4. Type of Study

- Randomized control trial

- Prospective cohort study

- Retrospective cohort study

\section{Diagnostic Certainty}

6. Description of the Surgical Procedure Given

7. Description of Postoperative Rehabilitation
- In all

- In $>80 \%$

- In $<80 \%$, not stated or unclear

- Adequate (technique stated and details for procedure given)

- Fair (technique stated without elaboration)

- Inadequate, not stated or unclear

- Well described with $>80 \%$ of subject returning

- Well described with $60-80 \%$ of subjects returning

- Protocol not reported or $<60-80 \%$ of subjects returning

Part B- Score may be given for each option in all three sections

8. Outcome Criteria

(If outcome criteria is vague, score is automatically 0 for section)

9. Procedure for Assessing Outcomes

10. Description of Subject Selection Process
- Outcome measures clearly defined

- Timing of outcome assessment clearly stated

- Use of outcome criteria that has reported good reliability

- Use of outcome with good sensitivity

- Subjects recruited (not taken from surgeons' files)

- Investigator independent of surgeon

- Written assessment

- Completion of assessment by subjects themselves with minimal investigator assistance

- Selection criteria reported and unbiased 5

- Recruitment rate reported- $>80 \%$ or $<80 \%$

- Eligible subjects not included in study satisfactorily accounted for or $100 \%$ recruitment 
Table D7. Dodson et al. ${ }^{21}$ Coleman Methodology Score

Part A- Only one score given for each section

1. Study Size (N)

- $>60$

Score

- $41-60$

$\underline{10}$

- $20-40$

- $<20$, not stated

- $>24$

- $12-24$

- $<12$, not stated or unclear

3. Number of different surgical procedures

- One surgical technique only included in each reported outcome. If more than one surgical technique is reported, separate outcomes for each should be reported

- More than one surgical technique, $>90 \%$ of subjects underwent same surgical technique

- Not stated, unclear or $<90 \%$ of subjects underwent same surgical technique

4. Type of Study

- Randomized control trial

- Prospective cohort study

- Retrospective cohort study

5. Diagnostic Certainty

6. Description of the Surgical Procedure Given

7. Description of Postoperative Rehabilitation
- In all

- In $>80 \%$

- In $<80 \%$, not stated or unclear

- Adequate (technique stated and details for procedure given)

- Fair (technique stated without elaboration)

- Inadequate, not stated or unclear

- Well described with $>80 \%$ of subject returning

- Well described with $60-80 \%$ of subjects returning

- Protocol not reported or $<60-80 \%$ of subjects returning

Part B- Score may be given for each option in all three sections

8. Outcome criteria

(If outcome criteria is vague, score is automatically 0 for section)

9. Procedure for Assessing Outcomes

10. Description of Subject Selection Process
- Outcome measures clearly defined

- Timing of outcome assessment clearly stated

- Use of outcome criteria that has reported good reliability

- Use of outcome with good sensitivity

- Subjects recruited (not taken from surgeons' files)

- Investigator independent of surgeon

- Written assessment

- Completion of assessment by subjects themselves with minimal investigator assistance

- Selection criteria reported and unbiased

- Recruitment rate reported- $>80 \%$ or $<80 \%$

- Eligible subjects not included in study satisfactorily accounted for or $100 \%$ recruitment $\underline{2}$ 
Table D8. Dugas et al. ${ }^{26}$ Coleman Methodology Score

Part A- Only one score given for each section

1. Study Size (N)

- $>60$

Score

- $41-60$

10

- $20-40$

- $<20$, not stated

2. Mean Follow-up (Months)

- $>24$

$\underline{5}$

- $12-24$

2

- $<12$, not stated or unclear

3. Number of different surgical procedures

- One surgical technique only included in each reported outcome. If more than one surgical technique is reported, separate outcomes for each should be reported

- More than one surgical technique, $>90 \%$ of subjects underwent same surgical technique

- Not stated, unclear or $<90 \%$ of subjects underwent same surgical technique

4. Type of Study

- Randomized control trial

- Prospective cohort study

- Retrospective cohort study

- In all

- In $>80 \%$

\section{Diagnostic Certainty}

- In $<80 \%$, not stated or unclear

6. Description of the Surgical Procedure Given

- Adequate (technique stated and details for procedure given)

- Fair (technique stated without elaboration) 3

- Inadequate, not stated or unclear

7. Description of Postoperative Rehabilitation

- Well described with $>80 \%$ of subject returning

- Well described with $60-80 \%$ of subjects returning

- Protocol not reported or $<60-80 \%$ of subjects returning

Part B- Score may be given for each option in all three sections

8. Outcome Criteria

(If outcome criteria is vague, score is automatically 0 for section)

9. Procedure for Assessing Outcomes

10. Description of Subject Selection Process
- Outcome measures clearly defined

- Timing of outcome assessment clearly stated

- Use of outcome criteria that has reported good reliability

- Use of outcome with good sensitivity

- Subjects recruited (not taken from surgeons' files) 5

- Investigator independent of surgeon 4

- Written assessment 3

- Completion of assessment by subjects themselves 3 with minimal investigator assistance

- Selection criteria reported and unbiased $\underline{\mathbf{5}}$

- Recruitment rate reported- $>80 \%$ or $<80 \% \quad 3$

- Eligible subjects not included in study satisfactorily accounted for or $100 \%$ recruitment $\underline{2}$ 
Table D9. Jobe et al. ${ }^{8}$ Coleman Methodology Score

Part A- Only one score given for each section

1. Study Size (N)

- $>60$

Score

- 41-60

10

- $20-40$

- $<20$, not stated

- $>24$

- $12-24$

- $<12$, not stated or unclear

3. Number of different surgical procedures

- One surgical technique only included in each reported outcome. If more than one surgical technique is reported, separate outcomes for each should be reported

- More than one surgical technique, $>90 \%$ of subjects underwent same surgical technique

- Not stated, unclear or $<90 \%$ of subjects underwent same surgical technique

4. Type of Study

- Randomized control trial

- Prospective cohort study

- Retrospective cohort study

5. Diagnostic Certainty

6. Description of the Surgical Procedure Given

7. Description of Postoperative Rehabilitation
- In all

- In $>80 \%$

- In $<80 \%$, not stated or unclear

- Adequate (technique stated and details for procedure given)

- Fair (technique stated without elaboration)

- Inadequate, not stated or unclear

- Well described with $>80 \%$ of subject returning

- Well described with $60-80 \%$ of subjects returning

- Protocol not reported or $<60-80 \%$ of subjects returning

Part B- Score may be given for each option in all three sections

8. Outcome Criteria

(If outcome criteria is vague, score is automatically 0 for section)

9. Procedure for Assessing Outcomes

10. Description of Subject Selection Process
- Outcome measures clearly defined

- Timing of outcome assessment clearly stated

- Use of outcome criteria that has reported good reliability

- Use of outcome with good sensitivity

- Subjects recruited (not taken from surgeons' files)

- Investigator independent of surgeon

- Written assessment

- Completion of assessment by subjects themselves with minimal investigator assistance

- Selection criteria reported and unbiased 5

- Recruitment rate reported- $>80 \%$ or 5 $<80 \%$

- Eligible subjects not included in study satisfactorily accounted for or $100 \%$ recruitment $\underline{2}$ 
Table D10. Koh et al..$^{19}$ Coleman Methodology Score

Part A- Only one score given for each section

1.Study Size (N)

- $>60$

Score

- $41-60$

10

- $20-40$

- $<20$, not stated

4

2. Mean Follow-up (Months)

- $>24$

$\underline{\mathbf{5}}$

- $12-24$

2

- $<12$, not stated or unclear

3. Number of different surgical procedures

- One surgical technique only included in each reported outcome. If more than one surgical technique is reported, separate outcomes for each should be reported

- More than one surgical technique, $>90 \%$ of subjects underwent same surgical technique

- Not stated, unclear or $<90 \%$ of subjects underwent same surgical technique

4. Type of Study

- Randomized control trial

- Prospective cohort study

- Retrospective cohort study

5. Diagnostic Certainty

6. Description of the Surgical Procedure Given

7. Description of Postoperative Rehabilitation
- In all

- In $>80 \%$

- In $<80 \%$, not stated or unclear

- Adequate (technique stated and details for procedure given)

- Fair (technique stated without elaboration)

- Inadequate, not stated or unclear

- Well described with $>80 \%$ of subject returning

- Well described with $60-80 \%$ of subjects returning

- Protocol not reported or $<60-80 \%$ of subjects returning

Part B- Score may be given for each option in all three sections

8. Outcome Criteria

(If outcome criteria is vague score is automatically 0 for section)

9. Procedure for Assessing Outcomes

10. Description of Subject Selection Process
- Outcome measures clearly defined

- Timing of outcome assessment clearly stated

- Use of outcome criteria that has reported good reliability

- Use of outcome with good sensitivity

- Subjects recruited (not taken from surgeons' files)

- Investigator independent of surgeon

- Written assessment

- Completion of assessment by subjects themselves with minimal investigator assistance

- Selection criteria reported and unbiased 5

- Recruitment rate reported- $>80 \%$ or $<80 \%$

- Eligible subjects not included in study satisfactorily accounted for or $100 \%$ recruitment $\underline{2}$

Total: 51/100 
Table D11. Merolla et al. ${ }^{27}$ Coleman Methodology Score

Part A- Only one score given for each section

1. Study Size (N)

- $>60$

Score

- 41-60

10

- $20-40$

- $<20$, not stated

- $>24$

- $12-24$

- $<12$, not stated or unclear

3. Number of different surgical procedures

- One surgical technique only included in each reported outcome. If more than one surgical technique is reported, separate outcomes for each should be reported

- More than one surgical technique, $>90 \%$ of subjects underwent same surgical technique

- Not stated, unclear or $<90 \%$ of subjects underwent same surgical technique

4. Type of Study

- Randomized control trial

- Prospective cohort study

- Retrospective cohort study

5. Diagnostic Certainty

6. Description of the Surgical Procedure Given

7. Description of Postoperative Rehabilitation
- In all

- In $>80 \%$

- In $<80 \%$, not stated or unclear

- Adequate (technique stated and details for procedure given)

- Fair (technique stated without elaboration)

- Inadequate, not stated or unclear

- Well described with $>80 \%$ of subject returning

- Well described with $60-80 \%$ of subjects returning

- Protocol not reported or $<60-80 \%$ of subjects returning

Part B- Score may be given for each option in all three sections

8. Outcome Criteria

(If outcome criteria is vague score is automatically 0 for section)

9. Procedure for Assessing Outcomes

10. Description of Subject Selection Process
- Outcome measures clearly defined

- Timing of outcome assessment clearly stated

- Use of outcome criteria that has reported good reliability

- Use of outcome with good sensitivity

- Subjects recruited (not taken from surgeons' files)

- Investigator independent of surgeon

- Written assessment

- Completion of assessment by subjects themselves with minimal investigator assistance

- Selection criteria reported and unbiased 5

- Recruitment rate reported- $>80 \%$ or $<80 \%$

- Eligible subjects not included in study satisfactorily accounted for or $100 \%$ recruitment
2 
Table D12. Osbahr et al. ${ }^{28}$ Coleman Methodology Score

Part A- Only one score given for each section

1.Study Size (N)

- $>60$

Score

- $41-60$

$\underline{10}$

- $20-40$

- $<20$, not stated

- $>24$

- $12-24$

- $<12$, not stated or unclear

3. Number of different surgical procedures

- One surgical technique only included in each reported outcome. If more than one surgical technique is reported, separate outcomes for each should be reported

- More than one surgical technique, $>90 \%$ of subjects underwent same surgical technique

- Not stated, unclear or $<90 \%$ of subjects underwent same surgical technique

4. Type of Study

- Randomized control trial

- Prospective cohort study

- Retrospective cohort study

\section{Diagnostic Certainty}

6. Description of the Surgical Procedure Given

7. Description of Postoperative Rehabilitation
- In all

- In $>80 \%$

- In $<80 \%$, not stated or unclear

- Adequate (technique stated and details for procedure given)

- Fair (technique stated without elaboration)

- Inadequate, not stated or unclear

- Well described with $>80 \%$ of subject returning

- Well described with $60-80 \%$ of subjects returning

- Protocol not reported or $<60-80 \%$ of subjects returning

Part B- Score may be given for each option in all three sections

8. Outcome Criteria

(If outcome criteria is vague score is automatically 0 for section)

9. Procedure for Assessing Outcomes

10. Description of Subject Selection Process
- Outcome measures clearly defined

- Timing of outcome assessment clearly stated

- Use of outcome criteria that has reported good reliability

- Use of outcome with good sensitivity

- Subjects recruited (not taken from surgeons' files)

- Investigator independent of surgeon

- Written assessment

- Completion of assessment by subjects themselves with minimal investigator assistance

- Selection criteria reported and unbiased 5

- Recruitment rate reported- $>80 \%$ or $<80 \%$

- Eligible subjects not included in study satisfactorily accounted for or $100 \%$ recruitment $\underline{2}$ 
Table D13. Paletta et al. ${ }^{29}$ Coleman Methodology Score

Part A- Only one score given for each section

1.Study Size (N)

- $>60$

Score

- $41-60$

10

- $20-40$

- $<20$, not stated

- $>24$

- $12-24$

- $<12$, not stated or unclear

3. Number of different surgical procedures

- One surgical technique only included in each reported outcome. If more than one surgical technique is reported, separate outcomes for each should be reported

- More than one surgical technique, $>90 \%$ of subjects underwent same surgical technique

- Not stated, unclear or $<90 \%$ of subjects underwent same surgical technique

4. Type of Study

- Randomized control trial

- Prospective cohort study

- Retrospective cohort study

5. Diagnostic Certainty

6. Description of the Surgical Procedure Given

7. Description of Postoperative Rehabilitation
- In all

- In $>80 \%$

- In $<80 \%$, not stated or unclear

- Adequate (technique stated and details for procedure given)

- Fair (technique stated without elaboration)

- Inadequate, not stated or unclear

- Well described with $>80 \%$ of subject returning

- Well described with $60-80 \%$ of subjects returning

- Protocol not reported or $<60-80 \%$ of subjects returning

Part B- Score may be given for each option in all three sections

8. Outcome Criteria

(If outcome criteria is vague score is automatically 0 for section)

9. Procedure for Assessing Outcomes

10. Description of Subject Selection Process
- Outcome measures clearly defined

- Timing of outcome assessment clearly stated

- Use of outcome criteria that has reported good reliability

- Use of outcome with good sensitivity

- Subjects recruited (not taken from surgeons' files)

- Investigator independent of surgeon

- Written assessment

- Completion of assessment by subjects themselves with minimal investigator assistance

- Selection criteria reported and unbiased 5

- Recruitment rate reported- $>80 \%$ or 5 $<80 \%$

- Eligible subjects not included in study satisfactorily accounted for or $100 \%$ recruitment 
Table D14. Rohrbough et al. ${ }^{22}$ Coleman Methodology Score

Part A- Only one score given for each section

1.Study Size (N)

- $>60$

Score

- $41-60$

10

- $20-40$

- $<20$, not stated

- $>24$

- $12-24$

- $<12$, not stated or unclear

3. Number of different surgical procedures

- One surgical technique only included in each reported outcome. If more than one surgical technique is reported, separate outcomes for each should be reported

- More than one surgical technique, $>90 \%$ of subjects underwent same surgical technique

- Not stated, unclear or $<90 \%$ of subjects underwent same surgical technique

4. Type of Study

- Randomized control trial

- Prospective cohort study

- Retrospective cohort study

5. Diagnostic Certainty

6. Description of the Surgical Procedure Given

7. Description of Postoperative Rehabilitation
- In all

- In $>80 \%$

- In $<80 \%$, not stated or unclear

- Adequate (technique stated and details for procedure given)

- Fair (technique stated without elaboration)

- Inadequate, not stated or unclear

- Well described with $>80 \%$ of subject returning

- Well described with $60-80 \%$ of subjects returning

- Protocol not reported or $<60-80 \%$ of subjects returning

Part B- Score may be given for each option in all three sections

8. Outcome Criteria

(If outcome criteria is vague score is automatically 0 for section)

9. Procedure for Assessing Outcomes

10. Description of Subject Selection Process
- Outcome measures clearly defined

- Timing of outcome assessment clearly stated

- Use of outcome criteria that has reported good reliability

- Use of outcome with good sensitivity

- Subjects recruited (not taken from surgeons' files)

- Investigator independent of surgeon

- Written assessment

- Completion of assessment by subjects themselves with minimal investigator assistance

- Selection criteria reported and unbiased 5

- Recruitment rate reported- $>80 \%$ or $<80 \%$

- Eligible subjects not included in study satisfactorily accounted for or $100 \%$ recruitment
2 
Table D15. Savoie et al..$^{30}$ Coleman Methodology Score

Part A- Only one score given for each section

1.Study Size (N)

- $>60$

Score

- $41-60$

$\underline{10}$

- $20-40$

- $<20$, not stated

- $>24$

- $12-24$

- $<12$, not stated or unclear

3. Number of different surgical procedures

- One surgical technique only

included in each reported outcome. If more than one surgical technique is reported, separate outcomes for each should be reported

- More than one surgical technique, $>90 \%$ of subjects underwent same surgical technique

- Not stated, unclear or $<90 \%$ of subjects underwent same surgical technique

4. Type of Study

- Randomized control trial

- Prospective cohort study

- Retrospective cohort study

\section{Diagnostic Certainty}

6. Description of the Surgical Procedure Given

7. Description of Postoperative Rehabilitation
- In all

- In $>80 \%$

- In $<80 \%$, not stated or unclear

- Adequate (technique stated and details for procedure given)

- Fair (technique stated without elaboration)

- Inadequate, not stated or unclear

- Well described with $>80 \%$ of subject returning

- Well described with $60-80 \%$ of subjects returning

- Protocol not reported or $<60-80 \%$ of subjects returning

Part B- Score may be given for each option in all three sections

8. Outcome Criteria

(If outcome criteria is vague score is automatically 0 for section)

9. Procedure for Assessing Outcomes

10. Description of Subject Selection Process
- Outcome measures clearly defined

- Timing of outcome assessment clearly stated

- Use of outcome criteria that has reported good reliability

- Use of outcome with good sensitivity

- Subjects recruited (not taken from surgeons' files)

- Investigator independent of surgeon

- Written assessment

- Completion of assessment by subjects themselves with minimal investigator assistance

- Selection criteria reported and unbiased $\underline{\mathbf{5}}$

- Recruitment rate reported- $>80 \%$ or $<80 \%$

- Eligible subjects not included in study satisfactorily accounted for or $100 \%$ recruitment
2

Total: 52/100 


\section{Figure D1. PRISMA Flow Diagram}

Google Scholar

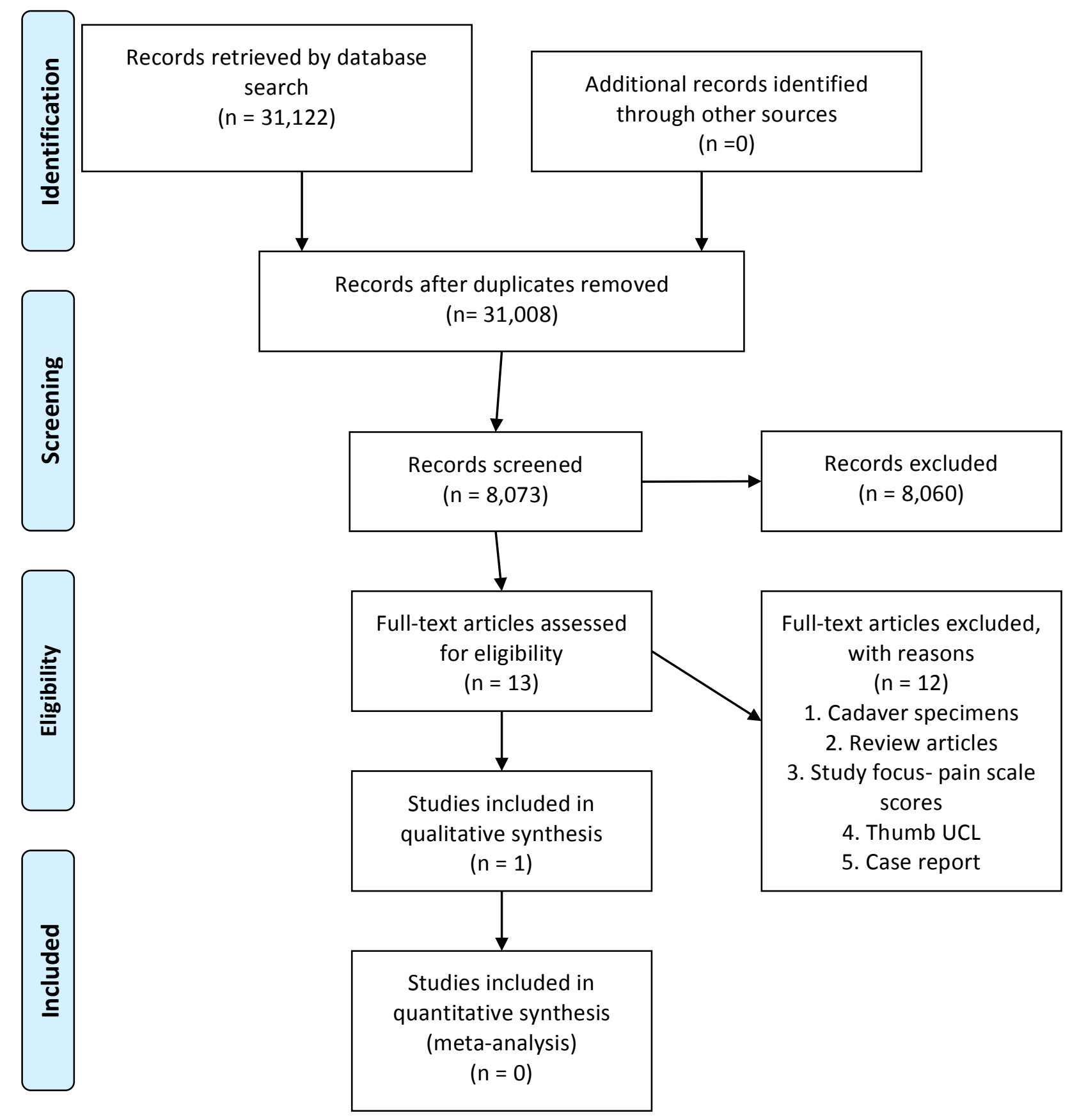


Figure D2. PRISMA Flow Diagram

ScienceDirect

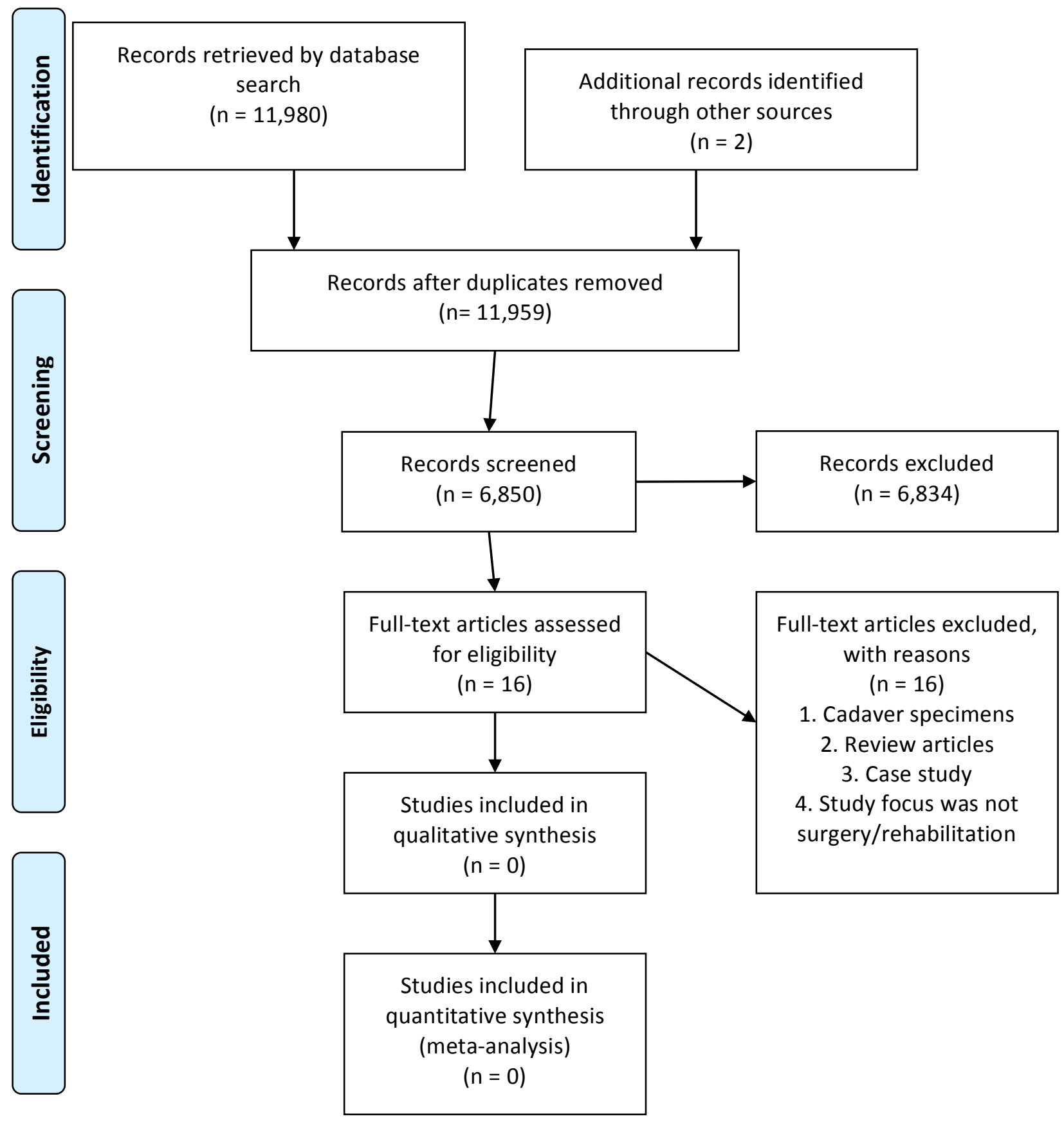


Figure D3. PRISMA Flow Diagram

CINAHL with full text

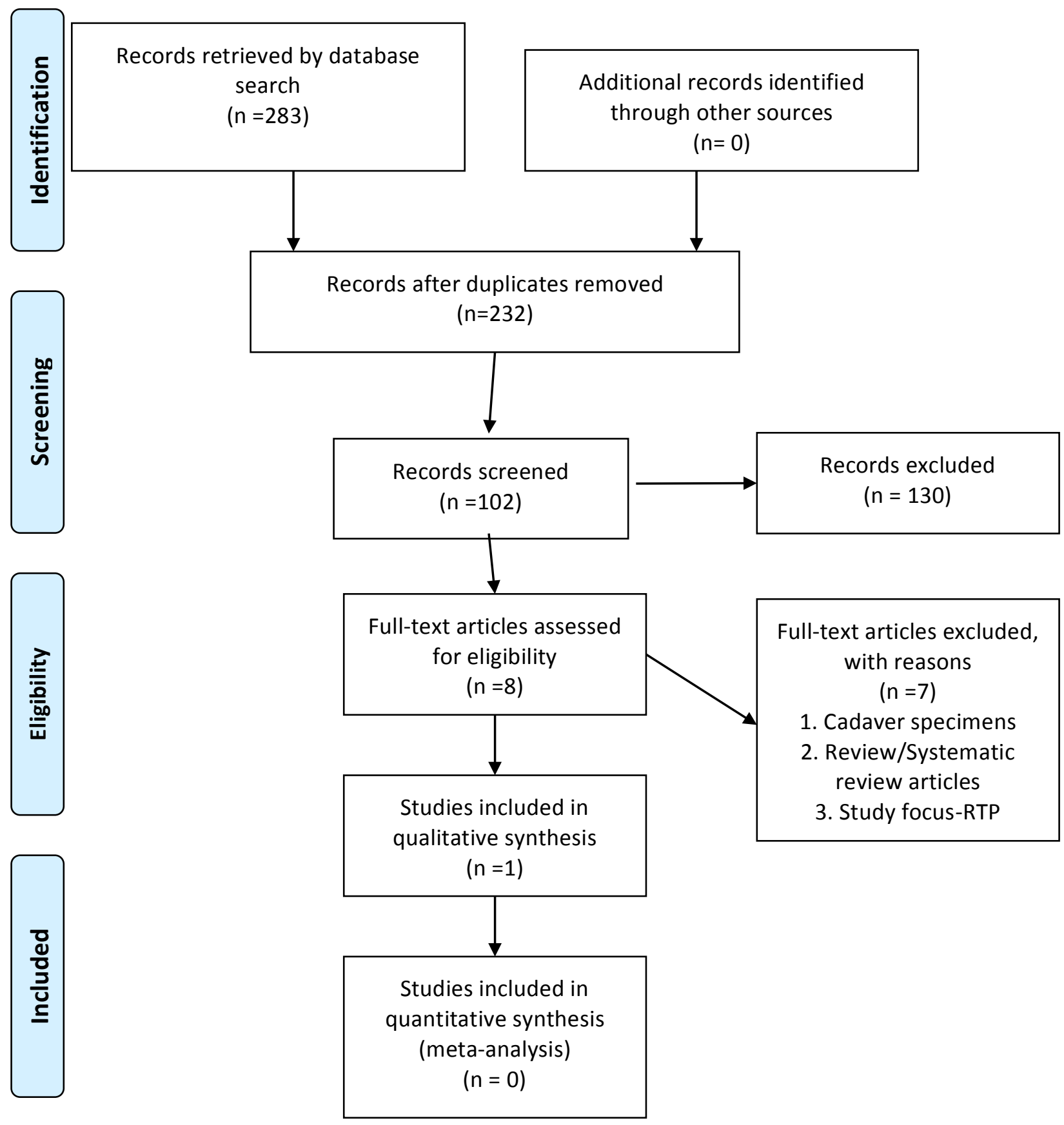


Figure D4. PRISMA Flow Diagram

MEDLINE (EbscoHost)

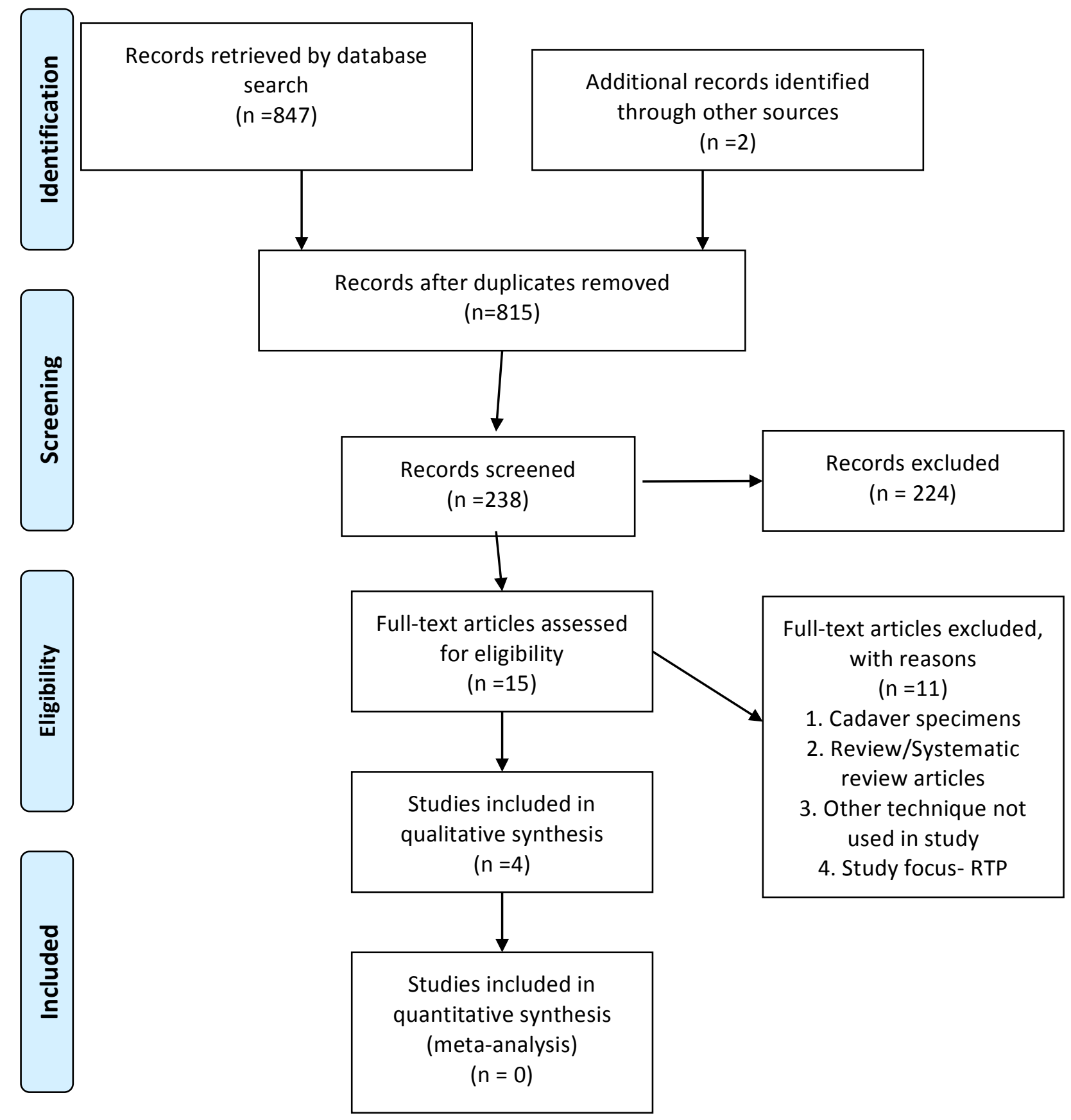


Figure D5. PRISMA Flow Diagram

MEDLINE (PubMed)

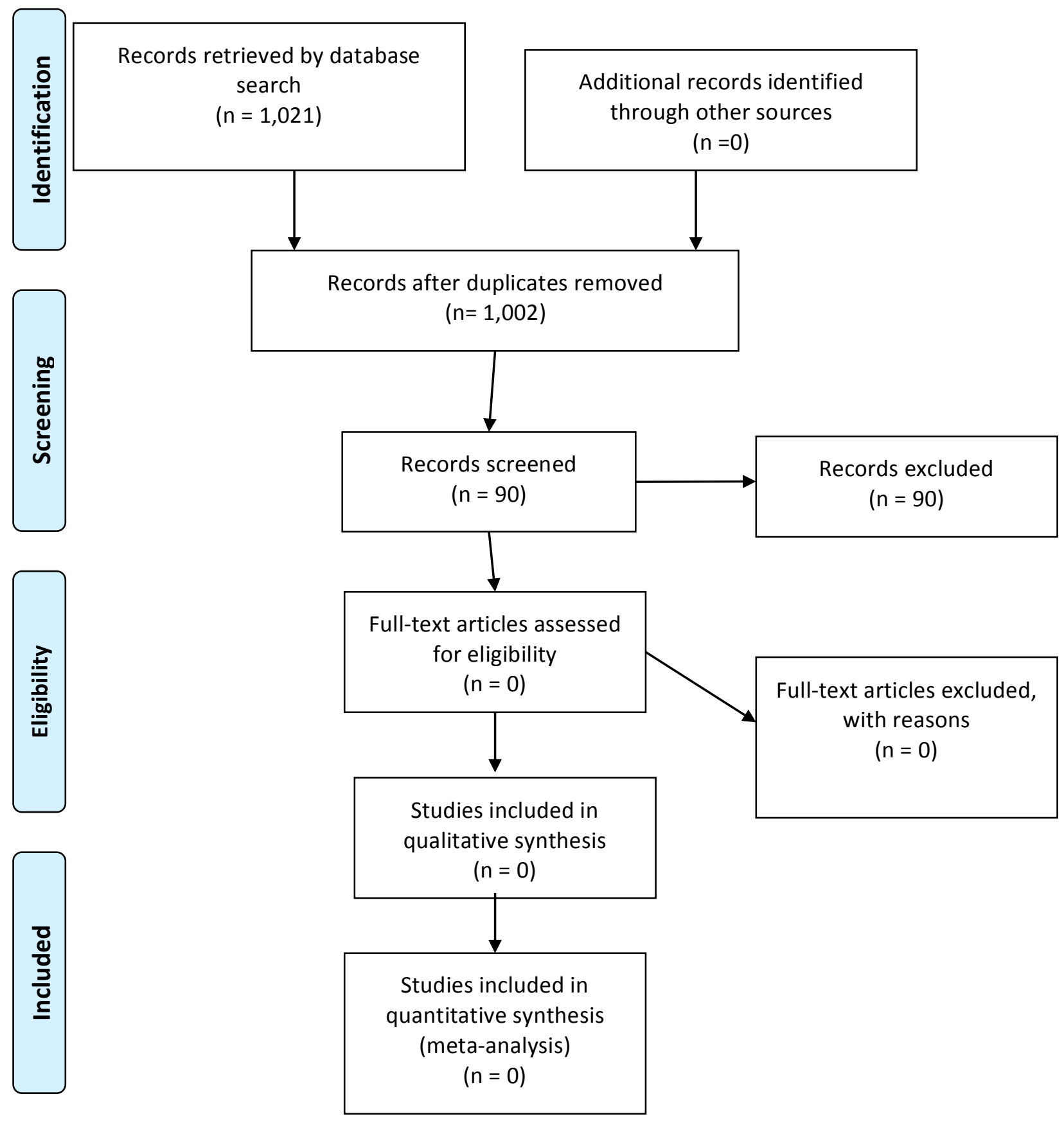




\section{Figure D6.PRISMA Flow Diagram}

SPORTDiscus

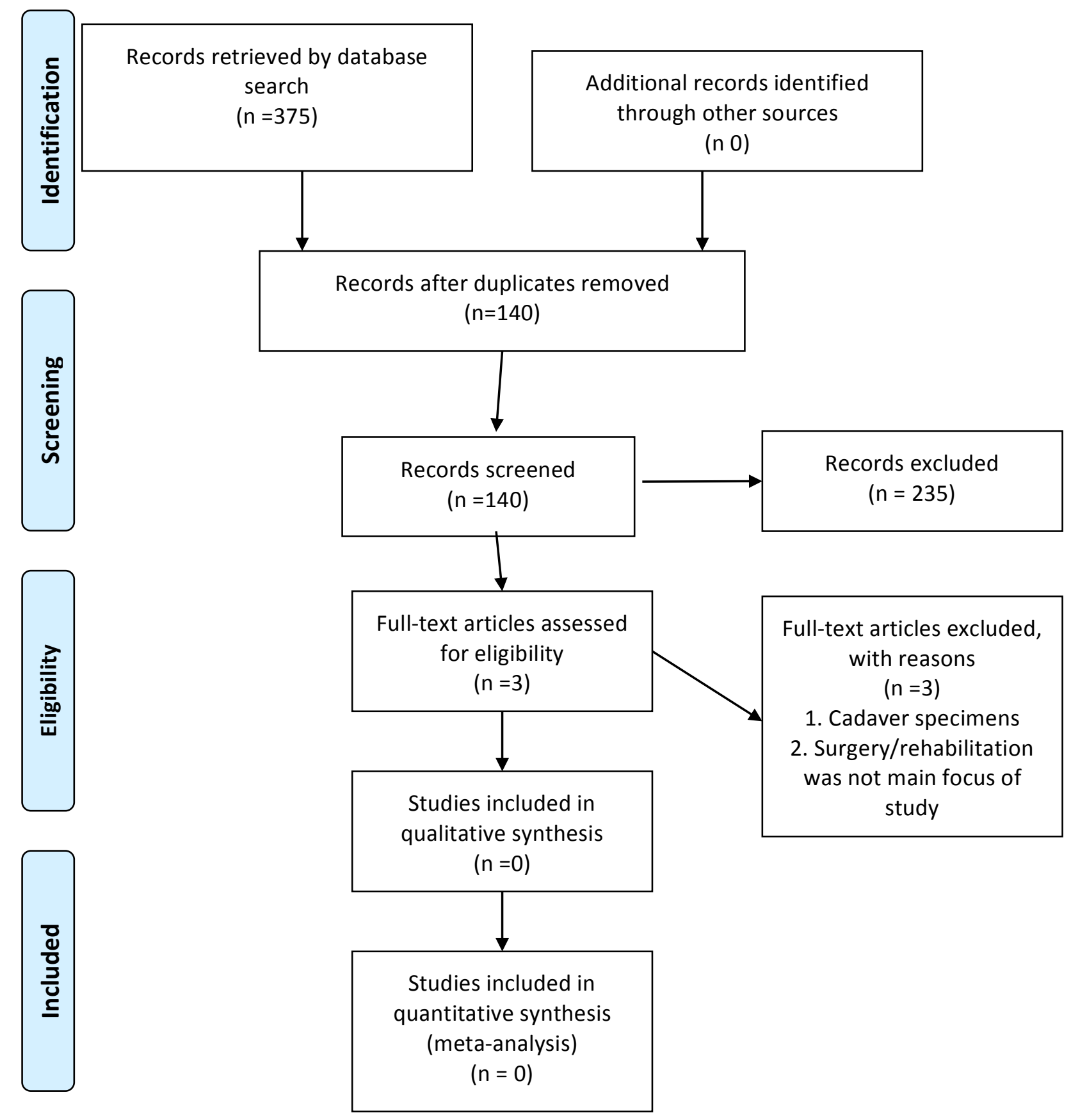


Figure D7. PRISMA Flow Diagram

PubMed

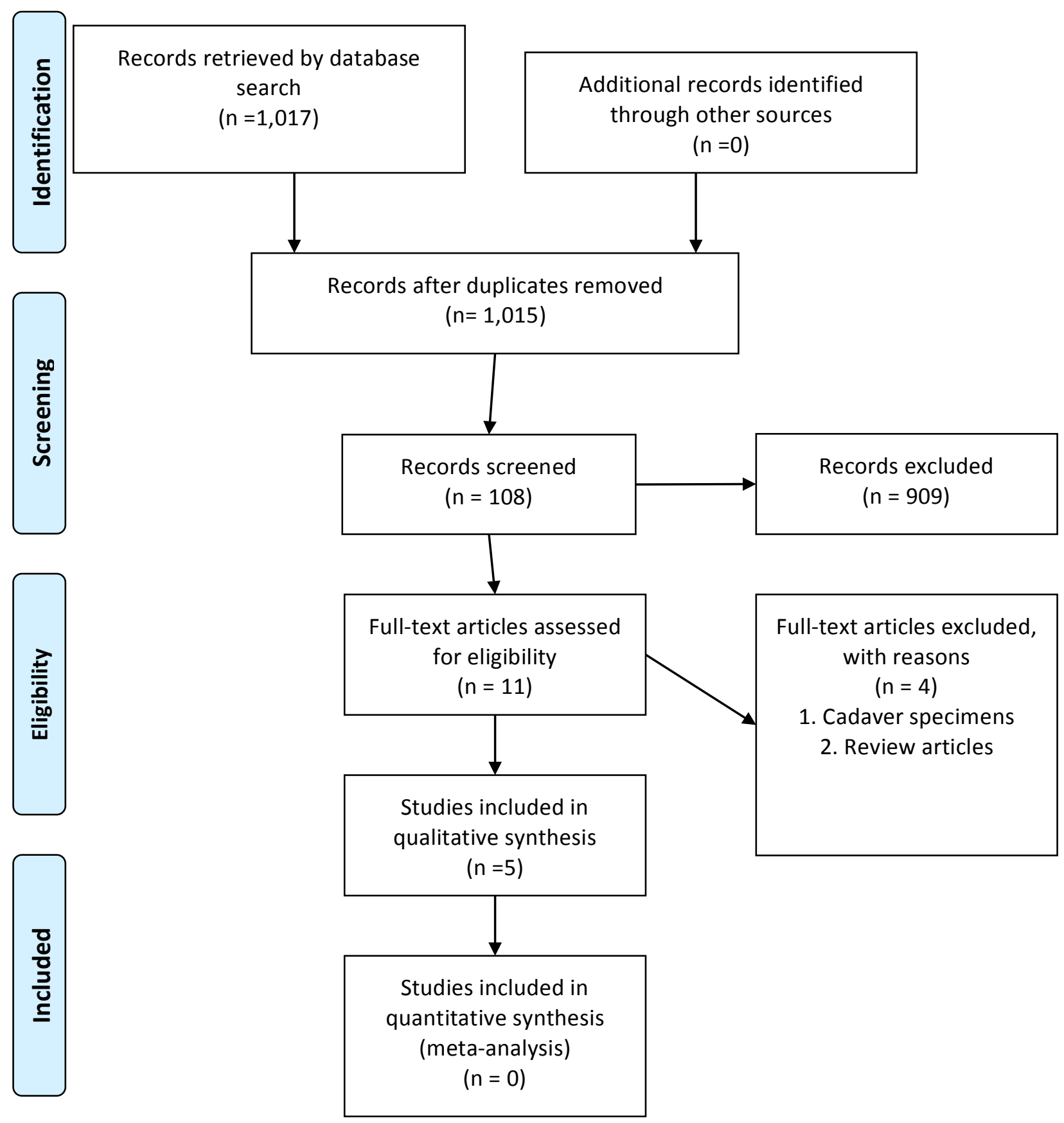


APPENDIX E

\section{RECCOMMENDATIONS FOR FUTURE RESEARCH}

1. Future studies could be expanded to cadaver studies and include a new research question on the anatomical and biomechanical aspect of a surgical technique.

2. Expand to studies that focus on reporting the use of more assessment outcomes and recruiting rates to increase the CMS scores.

3. Surgical studies should continue to use more objective outcome measures and report on effect sizes.

4. Find studies that were randomized control trials, or of higher level of evidence, with multiple follow-ups reported and outcome measures. 


\section{ADDITIONAL REFERENCES}

32. Keller RA, Steffes MJ, Zhuo, D, Bey MJ, Moutzouros V. The effects of medial ulnar collateral ligament reconstruction on major league pitching performance. J Shoulder Elbow Surg. 2014; 2014:1-8.

33. Paletta GA, Klepps SJ, Difelice GS, Allen T, Brodt MD, Burns ME, Silva MJ, Wright RW. Biomechanical evaluation of 2 techniques for ulnar collateral ligament reconstruction of the elbow. Am J Sport Med. 2006;34(10):1599-1603.

34. Prentice WE. Principles of athletic training: a competency-based approach. $14^{\text {th }}$ edition. New York, NY: McGraw-Hill Companies; 2011:681-699.

35. Nguyen D. Anatomy, Examination, and Imaging of the shoulder. Oper Tech Orthop. 2008;18(1):2-8.

36. Gordis L. Prospective cohort study design. Can Nurse. 2014;110(2):14.

37. Poehling GG, Jenkins CB. Levels of evidence and your therapeutic study- what's the difference with cohorts, controls, and cases? Arthroscopy. 2004;20(6):563.

38. Hernon P, Schwartz C. Reliability and validity. Libr Inf Sci Res. 2009;31(2):73-74.

39. McClure P. Sensitivity and specificity. J Hand Ther. 2001;14(3):219-220.

40. Bryce CD, Armstrong AD. Anatomy and biomechanics of the elbow. Orthop Clin N Am. 2008;39:141-154.

41. Makhni EC, Lee RW, Morrow ZS, Gualtieri AP, Gorroochum P, Ahmad CS. Performance return to competition and reinjury after Tommy John surgery in major league baseball pitchers. Am J Sport Med. 2014;42(6):1323-1332

42. Jones KJ, Conte S, Patterson N, ElAttrache NS, Dines JS. Functional outcomes following revision ulnar collateral ligament reconstruction in major league baseball. J Shoulder elbow. 2013;22:642-646.

43. Erickson BJ, Gupta AK, Harris JD, Bush-Joseph C, Bach BR, Abrams GD, San Juan AM, Cole BJ, Romeo AA. Rate of return to pitching and performance after Tommy John surgery in major leagues baseball pitchers. Am J Sport Med. 2013;42(3):536-543. 